Accepted for publichtion in the Astrophysical Journal: June 18, 2006

Preprint typeset using $\mathrm{LAT}_{\mathrm{E} X}$ style emulateapj v. 6/22/04

\title{
MULTIWAVELENGTH MONITORING OF THE DWARF SEYFERT 1 GALAXY NGC 4395. III. OPTICAL VARIABILITY AND X-RAY/UV/OPTICAL CORRELATIONS.
}

Louis-Benoit Desroches $^{1}$, Alexei V. Filippenko ${ }^{1}$, Shai Kaspi ${ }^{2,3}$, Ari Laor ${ }^{2}$, Dan Maoz ${ }^{3}$, Mohan Ganeshalingam ${ }^{1}$, Weidong Li ${ }^{1}$, Edward C. Moran ${ }^{4}$, Brandon Swift ${ }^{1}$, Misty C. Bentz ${ }^{5}$, Luis C. Ho ${ }^{6}$, Kirpal Nandra ${ }^{7}$, Paul M. O'Neill $^{7}$, AND BRAdley M. PETERSON ${ }^{5}$

Accepted for publication in the Astrophysical Journal: June 18, 2006

\begin{abstract}
We present optical observations of the low-luminosity Seyfert 1 nucleus of NGC 4395, as part of a multiwavelength reverberation-mapping program. Observations were carried out over two nights in 2004 April at Lick, Wise, and Kitt Peak Observatories. We obtained $V$-band and $B$-band photometry, and spectra over the range 3500-6800 A. Simultaneous Hubble Space Telescope UV and Chandra Xray observations are presented in companion papers. Even though NGC 4395 was in an extremely low state of activity, we detect significant continuum variability of $2-10 \%$, increasing toward shorter wavelengths. The continuum light curves, both spectroscopic and photometric, are qualitatively similar to the simultaneous UV and X-ray light curves. Inter-band cross-correlations suggest that the optical continuum emission lags behind the UV continuum emission by $24_{-9}^{+7} \mathrm{~min}$, and that the optical continuum emission lags behind the X-ray continuum emission by $44 \pm 13 \mathrm{~min}$, consistent with a reprocessing model for active galactic nucleus emission. There are also hints of Balmer emission lines lagging behind the optical continuum by an amount slightly larger than the emission-line lag detected in the UV. These results are all similar to those of other Seyfert 1 nuclei. The emission-line lag yields a mass measurement of the central black hole, which although not very significant, is consistent with the value derived from the simultaneous UV data.

Subject headings: galaxies: active — galaxies: individual (NGC 4395) — galaxies: nuclei — galaxies: Seyfert
\end{abstract}

\section{INTRODUCTION}

The nucleus of NGC 4395 is a "dwarf" type 1 Seyfert active galactic nucleus (AGN), the least luminous known in its class (Filippenko \& Sargent 1989; Filippenko et al. 1993). Its presence in an essentially bulgeless, extremely late-type galaxy is surprising; such AGNs (and quiescent black holes) are usually found in bulge-dominated earlytype systems (Ho et al. 1997), with black hole mass and bulge mass strongly correlated (Magorrian et al. 1998; Laor 1998; Marconi \& Hunt 2003; Häring \& Rix 2004). Despite this, the nucleus of NGC 4395 exhibits all of the hallmarks of a type 1 AGN: it is detected as a variable X-ray point source (Lira et al. 1999; Moran et al. 1999, 2005; Iwasawa et al. 2000; Ho et al. 2001; Shih et al. 2003), it has a high brightness temperature, highly compact radio core (Moran et al. 1999; Ho \& Ulvestad 2001; Wrobel et al. 2001), and its spectral energy distribution

\footnotetext{
1 Department of Astronomy, University of California, Berkeley, CA 94720-3411; louis@astro.berkeley.edu, alex@astro.berkeley.edu, mganesh@astro.berkeley.edu, wli@astro.berkeley.edu, bswift@ugastro.berkeley.edu

2 Department of Physics, Technion, Haifa 32000, Israel; laor@physics.technion.ac.il

3 Wise Observatory and School of Physics and Astronomy, Tel-Aviv University, Tel-Aviv 69978, Israel; shai@wise.tau.ac.il, dani@wise.tau.ac.il

4 Department of Astronomy, Wesleyan University, Van Vleck Observatory, Middletown, CT 06459; ecm@astro.wesleyan.edu

5 Department of Astronomy, Ohio State University, 140 West 18th Ave., Columbus, OH 43210-1173; bentz@astronomy.ohiostate.edu, peterson@astronomy.ohio-state.edu

6 Carnegie Observatories, 813 Santa Barbara St., Pasadena, CA 91101; lho@ociw.edu

7 Astrophysics Group, Imperial College London, Blackett Laboratory, Prince Consort Road, London, SW7 2AZ, UK k.nandra@imperial.ac.uk, p.oneill@imperial.ac.uk
}

from X-ray to radio wavelengths is similar to that of other type 1 AGNs (Moran et al. 1999).

NGC 4395 offers a chance to probe the low end of the supermassive black hole (SMBH) mass distribution. Knowing the mass of the central object will help constrain the $M_{\mathrm{BH}}-\sigma_{*}$ relation (Gebhardt et al. 2000; Ferrarese et al. 2001; Tremaine et al.|2002), which suggests a strong link between the growth of the $\mathrm{SMBH}$ and evolution of the host galaxy. The SMBH mass can be measured through emission-line reverberation mapping (Blandford \& McKee 1982; Peterson 1993; Netzer \& Peterson 1997). The relations of Kaspi et al. (2000, 2005) between continuum luminosity and radius of the broad-line region (BLR) suggest that the size of the Balmer line-emitting region in NGC 4395 is of order light hours.

We have used the reverberation method successfully in the ultraviolet (UV) with the Hubble Space Telescope $(H S T)$ to determine the size of the BLR and the mass of the central object, measured to be $M_{\mathrm{BH}}=$ $(3.6 \pm 1.1) \times 10^{5} M_{\odot}$ (Peterson et al. 2005, hereafter Paper I). We present here simultaneous optical observations that complement the UV results. Simultaneous X-ray observations with Chandra are presented by O'Neill et al. (2006, hereafter Paper II). The observations and data reduction are outlined in Section 2 while the data analysis is described in Section 3. In Section 4 we compare our results with those published for other AGNs and estimate the SMBH mass. We summarize our results in Section 5

\section{OBSERVATIONS AND REDUCTIONS}

\subsection{Lick Observatory}


Spectra were obtained on 2004 April 10 and 11 (UT dates are used throughout this paper) at the Lick Observatory 3-m Shane telescope, using the Kast spectrograph (Miller \& Stone 1993). The instrument separates blue and red light using a dichroic beamsplitter onto two $1200 \times 400$ pixel CCDs (optimized for blue or red light) for simultaneous coverage. The pixel scale in the crossdispersive direction is about 0.8 pixel $^{-1}$. The blue-side grism gave a resolution of $1.83 \AA$ pixel $^{-1}$ with an effective range of $3200-5400 \AA$, while the red-side grating was set to give a resolution of $1.17 \AA$ pixel $^{-1}$ with an effective range of 5300-6800 . The slit width was $2^{\prime \prime}$. Sequences of 5 to 8 exposures of NGC 4395, each of duration $10 \mathrm{~min}$, were bracketed by exposures of standard stars (HZ44 and Feige 34; Oke 1990). The position angle (PA) of the slit was set to the average parallactic angle during each sequence to minimize differential light loss (Filippenko 1982).

With BLR variability expected on sub-hour timescales, 10-min exposures were necessary to maximize temporal coverage while maintaining a reasonable signal-to-noise ratio $(\mathrm{S} / \mathrm{N})$. The standard stars were used to monitor atmospheric extinction and obtain relative flux calibration; absolute flux calibration of individual NGC 4395 spectra was done via narrow emission lines (expected to be constant in an AGN). The weather was generally clear, though not photometric, with occasional thin clouds. Conditions worsened slightly on the second night, with more cirrus clouds. Typical seeing conditions were variable and ranged from $1^{\prime \prime}$ to $2^{\prime \prime} .5$ full width at halfmaximum intensity (FWHM).

Spectra were bias subtracted, flat fielded, and wavelength calibrated with standard $\mathrm{IRAF}^{8}$ routines. Wavelength calibration on the blue side was achieved with a $\mathrm{HeHgCd}$ lamp; the red side was calibrated with a $\mathrm{NeAr}$ lamp. The extraction aperture was $4^{\prime \prime}$ along the crossdispersive axis. Spectra were then flux calibrated and extinction corrected with a standard star closely matching in airmass and time.

On April 11 there was an electronic irregularity with the red-side CCD. Several exposures were highly contaminated with a banding structure (i.e., entire pixel rows were forced to the same count level). This was eventually attributed to a loose connection inside the detector resulting in variable voltage. Consequently, several redside exposures on this night had to be discarded. Unfortunately, it is likely that this irregularity was present in most exposures from both nights, albeit not at a level severe enough to notice readily. We touch upon this issue again in Section 4.1

We also obtained simultaneous $B$-band observations with the 1-m Nickel telescope and $V$-band observations with the 0.76-m Katzman Automatic Imaging Telescope (KAIT; Filippenko et al. 2001) on both nights. The Nickel CCD was used in $2 \times 2$ binned mode, resulting in a $1024 \times 1024$ pixel detector with a pixel scale of $0{ }^{\prime \prime} 37$ pixel $^{-1}$, along with $5 \mathrm{~min}$ exposures. The Nickel data were bias subtracted automatically and flat fielded using standard IRAF routines. KAIT uses a back-illuminated

\footnotetext{
8 IRAF is distributed by the National Optical Astronomy Observatories, which are operated by the Association of Universities for Research in Astronomy, Inc., under cooperative agreement with the National Science Foundation.
}

Apogee $500 \times 500$ pixel CCD with a $0{ }^{\prime \prime} 8$ pixel $^{-1}$ scale. We used $4 \mathrm{~min}$ exposures. The KAIT data were reduced automatically as described by Li et al. (2001).

\subsection{Wise Observatory}

$V$-band observations were obtained on 2004 April 9-10 and 10-11, and on 2004 July 3, with the Wise Observatory 1-m telescope in Israel, using a back-illuminated Tektronix $1024 \times 1024$ pixel CCD. The image scale was 0.7 pixel $^{-1}$. The nucleus was positioned on the northwest corner of the chip to maximize the number of relatively bright reference stars included in the field. Exposures of $262 \mathrm{~s}$ each were obtained about once per 5 min. One exposure in the $B$ band was also obtained for the purpose of measuring the $B-V$ colors of the stars in the field. Observations were carried out each night from the start of the night until the airmass of NGC 4395 exceeded $\sim 2$. Typical image quality was $2-3^{\prime \prime}$ FWHM, deteriorating toward the end of each night at high airmass. Standard debiasing and flat fielding were performed.

\subsection{Kitt Peak National Observatory}

Spectroscopic observations at the KPNO Mayall 4-m telescope were obtained on 2004 April 10 and 11 . We used the Ritchey-Chrétien spectrograph with the KPC17B grating (527 $\mathrm{l} \mathrm{mm}^{-1}$ ) and the TEK2KB detector. Observations with this setup covered the range of about 4100-7300 $\AA$ with a resolution of $4.2 \AA$. We obtained only 6 and 16 exposures of 5 min duration on April 10 and 11 , respectively, a result of rain. We elect to present only the April 11 data, which consist of a longer, nearly uninterrupted span of observations. The weather for the April 11 data was clear (nearly photometric) until dawn.

The observation and calibration method we used is the same as described by Maoz et al. (1994) and Kaspi et al. (2000). This method achieves spectrophotometric calibration by rotating the long spectrograph slit to the appropriate position angle (PA) so that a nearby comparison star is observed simultaneously with the object. The comparison star we used is located at a $\mathrm{PA}$ of $51^{\circ}$ relative to the nucleus of NGC 4395 and at a distance of $33^{\prime \prime}$ (star 1 in Figure 1). We used a slit width of $2^{\prime \prime}$. This PA is only $10^{\circ}$ off the parallactic angle for this object at high airmass $(\sim 2)$, and since the meridian transit occurs near the zenith, atmospheric dispersion losses for most of the night are small. We also used the atmospheric dispersion corrector (i.e., Risley prisms) to minimize the dispersion losses at this nonparallactic position angle. We expand upon this issue in Section 3.2

The spectroscopic data were reduced using standard IRAF routines, and for each exposure we obtained an AGN/star flux-ratio spectrum. These spectra were compared to each other to enable cleaning of cosmic-ray events. The spectra were calibrated to an absolute flux level using observations of the spectrophotometric standard star HZ44 taken adjacent to the observations of NGC 4395. Although the absolute flux calibration has an uncertainty of $\sim 10 \%$, the relative calibration accuracy between the observations is about $1 \%$ (confirmed using the [O III] line fluxes).

\section{DATA ANALYSIS}

\subsection{Photometry}


For the KAIT and Nickel data, we examined the images to determine a FWHM for each night of observations, and found it to be roughly constant at $\sim 4$ pixels $\left(\sim 3^{\prime \prime}\right)$ for KAIT and $\sim 8$ pixels $\left(\sim 3^{\prime \prime}\right)$ for Nickel. We chose an aperture with a radius set to this size and performed aperture photometry on the nucleus and nearby reference (comparison) stars. The sky was subtracted using a 5-pixel wide annulus with an inner radius 20 pixels from the center. We also attempted point-spread function (PSF) photometry, but found the results very similar with a slight increase in noise, possibly due to the relatively faint reference stars, and decided to proceed with aperture photometry instead. To correct for extinction, non-photometric conditions, and variable seeing, we normalized the NGC 4395 flux to those of stars 1 and 2 (see Figure (1). These stars were chosen for their proximity to the nucleus, their similar $B-V$ colors, and overlap with the reference stars used in the analysis of the Wise data. Because of the relative photometry, the uncertainties in the photometry were estimated using the observed scatter of the reference stars.

For the Wise data, we chose five stars, all brighter than the nucleus (by 0.4 to $3.6 \mathrm{mag}$ in $V$ ), with $B-V$ colors similar to that of the nucleus (from $0.1 \mathrm{mag}$ bluer to 0.3 mag redder), to serve as photometric reference stars (see Figure (1). Aperture photometry within a fixed, 5-pixel $(3 . \prime 5)$ radius aperture was performed on the nucleus and on the stars, and the background level was determined and subtracted using a 5-pixel wide annulus separated by a 4-pixel interval from the central aperture. This separation is sufficient to exclude the wings of the PSF in the sky annulus.

The photometric zero point of every exposure was found by taking, for each star, the instrumental magnitude difference between the exposure and the first epoch of the night, and calculating the mean difference for all five stars. The light curve of each star, relative to the zero point determined by the remaining four stars, was examined and verified to be constant, and its root-meansquare (rms) fluctuations were noted; these provide an empirical estimate of the accuracy of the photometry. Outlier points in each star's light curve were then excluded. The mean of each star's magnitude was subtracted from its light curve, to avoid any dependence on the zero point of the flux at the first epoch. Finally, the photometric zero point based on the five stars was recalculated, and used to calibrate the measurements of the nucleus.

We used the sum of the fluxes from the common reference stars 1 and 2 (Figure 1) to normalize the light curves from all three telescopes. Photometric accuracy, based on the reference stars, is generally better than $1-2 \%$, except toward the end of each night, when the high airmass starts to have an effect. To obtain an absolute flux scale, we used the Sloan Digital Sky Survey SkyServer ${ }^{9}$ to retrieve $g$ and $r$ magnitudes for stars 1 and 2 . The corresponding calibrated $B$ and $V$ magnitudes were then found using the transformations of Smith et al. (2002).

Figures 2 and 3 show the various light curves of the NGC 4395 nucleus (reproduced in Table1) and the reference stars. Intra-night variability was greatest during the first night of Wise observations. Table 2 lists variability

\footnotetext{
${ }^{9}$ http://cas.sdss.org/astro/en/
}

parameters for these light curves. The excess variance $F_{\text {var }}$ is as described by Rodriguez-Pascual et al. (1997) and represents the measured variance above the noise, $R_{\text {max }}$ is the maximum-to-minimum flux ratio, and $A_{\text {peak }}$ is simply the peak-to-valley amplitude divided by the unweighted mean of the night. On April 10, NGC 4395 was observed for 1.5 hours longer from KAIT than from the Nickel telescope, when there was a sharp decline in brightness. It is for this reason that KAIT exhibits a greater $F_{\text {var }}$ on April 10 than Nickel, even though variability is generally more pronounced toward bluer colors for typical Seyferts. April 11, with equal KAIT/Nickel coverage, is a better indicator of relative $V$-band and $B$-band variability.

Figure 3 shows the 2004 July 3 observations (corresponding to HST Visit 3 from Paper I) from Wise Observatory. NGC 4395 was only observed for 2 hours, before it was too low in the sky. Consequently, it is unclear whether the low variation amplitude is intrinsic or the result of the short observing window. The nucleus was a factor of about 1.5 brighter in the $V$ band in July, to be compared to a factor of 3.4 in the UV (Paper I). In terms of the higher variation amplitude at shorter wavelengths during this time of increased activity, NGC 4395 is once again behaving like a typical AGN (see Section 4.1).

\subsection{Spectroscopy}

The Lick spectra were grouped according to blue/red side and night. Within each group, the spectra were cross-correlated against each other and rebinned to correct for slight wavelength solution discrepancies, to an accuracy of $0.1 \AA$. The spectra were then flux normalized according to strong narrow emission lines: [O III] $\lambda \lambda 4959,5007$ on the blue side, the sum of [S II] $\lambda \lambda 6716,6731$ and [O I] $\lambda 6300$ (equally weighted) on the red side. Since the red and blue sides have a very small overlap region, which is compromised by dichroic light loss, it was not possible to calibrate the red side to the blue side. To remedy this, we used complete KPNO spectra to determine a mean $[\mathrm{O} \mathrm{III}] /([\mathrm{S} \mathrm{II}]+[\mathrm{O} \mathrm{I}])$ ratio, and applied this to the Lick spectra to calibrate the red side to the blue side. The narrow-line flux normalization provided good relative calibration, but the absolute flux calibration of KPNO data was superior. We therefore used the [O III] flux to adjust the Lick data to the absolute scale of the KPNO data.

We then obtained flux measurements of $\mathrm{H} \alpha, \mathrm{H} \beta, \mathrm{H} \gamma$, He II $\lambda 4686$, and the individual [O III] lines as a means to examine the behavior of the spectra and the scatter of the narrow lines. The resolution on the blue side is sufficient to completely separate both [O III] lines. The continuum for each line was determined by averaging the continuum on either side of the line. Each line is well separated and the continuum is very flat over such a short range in wavelength. All flux measurements, including those of narrow lines, were simple integrations from continuumsubtracted spectra. Note that $\mathrm{H} \alpha$ also includes [N II], a narrow and non-varying line. Continuum measurements were obtained at $3650,4200,4550,5100,5625,6200$, and $6410 \AA$. They are median values over a small window typically $100 \AA$ wide, except for $5100 \AA$ which is $80 \AA$ wide, $5625 \AA$ which is $50 \AA$ wide, and $6410 \AA$ which is 40 $\AA$ wide. These windows have been verified to be featureless in a high $\mathrm{S} / \mathrm{N}$ coadded spectrum, and are as large as 
possible to reduce the noise. Despite this, the continuum light curves remain very noisy, especially towards shorter wavelengths, due to the low overall flux level.

Standard $1 \sigma$ errors are calculated for each flux measurement (which include Poisson, readout noise, sky, flatfielding, and calibration errors). Since all spectra are normalized via narrow lines, which are assumed to be constant, there is potential for extra systematic errors. Indeed, we find the rms scatter of [O III], [O II], [O I], and $[\mathrm{S} \mathrm{II}]$ to be about 3 times greater than the formal $1 \sigma$ errors, and so we adjust our measured errors by this factor to represent the true $1 \sigma$ uncertainty in the data.

For consistency, we repeated the above line and continuum flux measurements with the KPNO data (except [O II] and $3650 \AA$, which are outside the wavelength coverage). Since those spectra were observed and reduced with a different strategy, this provided a good check on all our measurements. Uncertainties were again estimated using the scatter of narrow lines, especially [O III].

Figures 4 and 5] show continuum light curves from the Lick and KPNO data, while Figures [6] and [7] show the measured light curves of emission lines. These are also reproduced in Tables 3 and 4 In order to decrease the noise, continuum measurements have been paired and averaged, with $3650 \AA$ omitted as it is very noisy. Overall, continuum variability on April 10 is greater at bluer wavelengths, as expected, while April 11 exhibits optical variability that is less strongly dependent on wavelength. The agreement between the Lick and KPNO continuum light curves, despite the different observing strategies, is reassuring.

The general consistency and stability of the narrow lines on April 10 indicates no obvious systematic effects, other than increased noise at higher airmass toward the end of the night. We note that [O I] and [S II] are mirror images of each other, since their sum was used to normalize the spectra. Similarly, [O III] $\lambda 4959$ (not shown) is a mirror image of [O III $\lambda 5007$. On April 11, the greater fluctuations of the narrow forbidden emission lines, especially on the red side ([O I] and $[\mathrm{S} \mathrm{II}])$, are likely a result of slightly compromised electronics. Weather was also generally worse than on April 10, resulting in lower $\mathrm{S} / \mathrm{N}$ data. KPNO measurements are included in Figure 7 for comparison, with overall agreement between the two sets of data. The major exception is the $\mathrm{H} \alpha$ light curve, which shows substantially more variability in the Lick data than in the KPNO data. Again, this is likely partially due to the electronics, with the Lick errors underestimated as a result.

Table 5 lists the variability parameters of the variable emission lines from the Lick data; we omit the KPNO data due to its short observing window. The narrow lines have $F_{\text {var }}=0$ by definition (except for [O II], which equals zero due to the noise), and exhibit rms scatter of $\sim 1 \%$ for $[\mathrm{O} \mathrm{III}], \sim 2 \%$ for $[\mathrm{O} \mathrm{I}]+[\mathrm{S} \mathrm{II}]$, and $\sim 6 \%$ for [O II]. For comparison, the variability parameters of 1350 $\AA$ (corresponding to $H S T$ Visit 2 on April 11, Paper I) and of the $0.4-8.0 \mathrm{keV} \mathrm{X}$-rays (corresponding to Chandra Visits 1 and 2 on April 10 and 11, Paper II) are also included. For the X-rays, we use $300 \mathrm{~s}$ bins and the full duration of each observation $(\sim 30 \mathrm{ks})$ in order to have similar time-scale ranges. We detect marginal variability in the lines of interest with broad components. The April
$11 \mathrm{H} \alpha$ variability is likely overestimated, again a result of the electronic difficulties. We detect significant continuum variability above the noise, which declines toward the red. This is consistent with photometric results from April 11 (with equal KAIT/Nickel coverage). The 3650 $\AA$ light curve is highly noisy, and no variability above the noise is detected on April 11.

It is possible that the non-parallactic KPNO observations (and subsequent Lick blue-side/red-side calibration using KPNO data) introduced a wavelength-dependent error, but we estimate such atmospheric dispersion losses to be minimal given the slit width and PA used. The use of Risley prisms also minimizes such dispersion losses. Inspection of the $[\mathrm{O} \mathrm{III}] /([\mathrm{S} \mathrm{II}]+[\mathrm{O} \mathrm{I}])$ ratio in the $\mathrm{KPNO}$ data revealed only a $\sim 4 \%$ discrepancy near the end of the observations, at high airmass. We did not use these data points, however, in obtaining a mean ratio to calibrate the blue and red sides of the Lick spectra. Even if dispersion-related errors persist, we note that this will not affect our measured $F_{\text {var }}$ values, as both the flux and flux error will be erroneous by the same factor. Only the relative scaling between the blue-side and red-side optical continua, seen in Figures 4 and 5 , could be affected by such errors.

We plot $F_{\text {var }}$ as a function of wavelength in Figure 8 We note that $F_{\text {var }}$ represents measured variability above the noise. For instance, the Lick continuum $F_{\text {var }}$ values with smaller windows (5100, 5625, and $6410 \AA$ ) are somewhat lower than their neighbors as a result of higher noise. Recall also that the Nickel data on April 10 span a shorter time than either the KAIT or the Lick 3-m data. What is clear from Figure 8 is the broad trend of increased variability with decreasing wavelength, the obviously lower variability on April 11, and the shallower dependence on wavelength on April 11.

These $F_{\text {var }}$ measurements are consistent with the general continuum light curve shapes from Figures 4 and 5 and with the broad trend in spectral slope (i.e., the slope of the continuum). It is quite clear that April 10 exhibits a steepening of the slope with increasing flux (and thus shorter wavelengths have greater variability), whereas on April 11 the slope is nearly constant and a weak function of total flux. This also agrees with the photometric $B-V$ (though on April 10, the Nickel data do not cover the sharp decline in flux at the end of the night, and therefore strong changes in $B-V$ cannot be seen). As the continuum light curves are very noisy, however, especially at short wavelengths, we do not attempt to characterize the spectral slope any further.

Figures 9] and 10] show a comparison between KAIT, Nickel, and Lick 3-m spectroscopic "broad-band" light curves. For this purpose, Lick spectra were passed through two filter functions and integrated in order to mimic $V$-band and $B$-band observations (Table 6). The sharp rise in UV luminosity on April 11 (Figure 1 from Paper I) is far more subdued in the $B$ and $V$ bands. There is overall agreement between the two $B$-band and $V$-band data sets to within $\sim 5-10 \%$. There exists an odd discrepancy, however, between the Lick spectroscopic "broad-band" light curves and the photometric light curves for a short period on both nights (JD-2453105, $0.78-0.85$ and $1.80-1.87$ ). This discrepancy is mirrored in the individual spectroscopic continuum light curves from 
Figures 4 and 5 These occur while NGC 4395 crosses the meridian, and correspond to a distinct subgroup of observations on both nights.

The discrepancy is not due to contamination from an $\mathrm{H}$ II region as the slit rotated over the nucleus, because extra line flux would cause a lower continuum with our normalization. Line intensity ratios, such as $[\mathrm{O} \mathrm{III}] / \mathrm{H} \beta$, also remain constant throughout the night. Galaxy contamination is not the source either, because (a) the galactic stellar light is very symmetrical about the nucleus, and (b) galactic stellar light is almost nonexistent in this bulgeless system (Filippenko \& Ho 2003). We also rereduced the suspect data with a different standard star (with similar airmass) and saw little change. Comparing the calibration coefficients between Lick and KAIT data (which have nearly identical weather) suggests that the narrow emission-line calibration is indeed at fault, and not extra continuum light.

The cause of this calibration anomaly is unknown. As noted previously, however, an electronic irregularity forced us to discard several red-side images on April 11. This problem occurred only near transit, when the position of the telescope was such as to cause the greatest disruption with the loose cable. Since the suspect data occur near transit, it is possible that this discrepancy is the result of a subtle electronic glitch affecting both CCDs, though this is only speculation. Whatever the cause, we caution the reader about any continuum measurements from the Lick data during these short periods of time.

\subsection{Cross-Correlations}

Our optical observations were carried out simultaneously with HST STIS observations as described in Paper I and with Chandra ACIS-S observations which are described in Paper II. Unfortunately, during the night of April 10, HST observations were acquired under gyro control which degraded the photometric accuracy, rendering these observations useless for our purposes. We used the April 11 observations along with the two Chandra observations for a cross-correlation analysis with the optical observations. The light curves used in this analysis are presented in Figure [1] We refer to the observations of 2004 April 10 and 11 as Visit 1 and Visit 2, respectively, to be consistent with Papers I and II.

In the following we use two cross-correlation methods: the interpolated cross-correlation function (ICCF; e.g., Gaskell \& Sparke 1986; Gaskell \& Peterson 1987; White \& Peterson 1994; Peterson et al. 2004) and the z-transform discrete correlation function (ZDCF) of Alexander (1997) which is an improvement over the discrete correlation function (DCF; Edelson \& Krolik 1988). ${ }^{10}$

To calculate the uncertainties in the cross-correlation lag determination we used the model-independent FR/RSS Monte Carlo method of Peterson et al. (1998, 2004, 2005). In this method, each Monte Carlo simulation consists of two parts. The first is a "random subset selection" (RSS) procedure which consists of randomly resampling a new light curve of $N$ points from the origi-

\footnotetext{
10 The ZDCF applies Fisher's $z$ transformation to the correlation coefficients, and uses equal population bins rather than the equal time bins used in the DCF.
}

nal light curve of $N$ points, without regard to whether a particular point has been selected previously. Thus some points are selected multiple times and others are not selected at all. The uncertainty of a single point that is selected $M$ times is decreased by a factor of $M^{1 / 2}$. The second part is "flux randomization" (FR) in which the observed fluxes are altered by random Gaussian deviates scaled to the uncertainty ascribed to each point.

The two resampled and altered time series are then cross-correlated using the ICCF method and the centroid of the CCF is computed. We used $\sim 10000$ Monte Carlo realizations to build up a cross-correlation centroid distribution (CCCD; e.g., Maoz \& Netzer 1989). The mean of the distribution is taken to be the time lag and the uncertainty is determined by the range which contains $68 \%$ of the Monte Carlo realizations in the CCCD and thus would correspond to $1 \sigma$ uncertainties for a normal distribution.

Cross-correlation functions of the emission-line light curves from Figures [6] and 7 with the $V$-band continuum light curve (Figure 11b) are presented in Figure 12 In each case, the emission-line and $V$-band light curves from both nights are combined into one. The crosscorrelation of the $\mathrm{H} \alpha$ line light curve yields a time lag of the line behind the continuum of $0.060_{-0.030}^{+0.034} \mathrm{~d}$ (Figure 12a), though $\sim 10 \%$ of the FR/RSS Monte Carlo simulations failed to yield successful centroid calculations as no well-defined peak was found. We note that the electronic difficulties on the red side exaggerate the $\mathrm{H} \alpha$ variations but leave the overall shape intact. The cross-correlation of the $\mathrm{H} \beta$ line light curve yields a very shallow peak using the ZDCF method and no peak at all when using the ICCF method (Figure 12b). Indeed $\sim 80 \%$ of the FR/RSS Monte Carlo simulations failed to yield successful centroid calculations. The $\sim 20 \%$ successful simulations yield a time lag of the line behind the continuum of $0.047_{-0.051}^{+0.050} \mathrm{~d}$. The cross-correlation the $\mathrm{H} \gamma$ line light curve has a more clearly defined peak than for $\mathrm{H} \beta$ though here also $\sim 40 \%$ of the FR/RSS Monte Carlo simulations failed to yield successful centroid calculations. The $\sim 60 \%$ successful simulations yield a time lag of the line behind the continuum of $0.059_{-0.060}^{+0.057} \mathrm{~d}$ (Figure 12:). The FR/RSS Monte Carlo simulations for the He II $\lambda 4686$ line failed in $\sim 70 \%$ cases, again indicating that the CCF does not have a well-defined peak. The $\sim 30 \%$ successful simulations yield a time lag of the line behind the continuum of $0.043_{-0.064}^{+0.069} \mathrm{~d}$ (Figure 12]).

We repeated the above cross-correlations with the emission-line light curves without the problem transit points described in Section 3.2 to check for any possible systematic errors. Our results were consistent, with any variation clearly within the noise of our simulations, and so we proceeded with our original cross-correlations above. This provides assurance that the problems associated with the anomalous continuum measurements during transit are not propagated to the emission-line light curves.

We note that the ICCFs and ZDCFs presented in Figure 12 are only loosely consistent with each other. In particular, the $\mathrm{H} \beta$ line (Figure 12 $\mathrm{b}$ ) exhibits the greatest difference between the two correlation methods. This arises because of the very small variation amplitude in the underlying light curves and the fact that there is a 
large gap between the two nights. This gap is interpolated over when using the ICCF method, which produces some extra correlation that distorts the ICCF from the ZDCF. It is reasonable to assume that in this case, the $\mathrm{ZDCF}$ is a better representation of the true CCF. Since the correlations and the time lags are of very low significance, however, and are only suggestive, we did not investigate this issue further. The CCFs in Figure 13 do not show this problem because the underlying light curves have higher variation amplitudes.

All CCFs presented in Figure 12 therefore have some, but not very significant, indication of a positive time lag behind the optical continuum. Although individual results are either consistent with zero or of low significance, the trend as a whole is quite suggestive. In particular, the average lag of the Balmer emission lines is about $0.056 \pm 0.047 \mathrm{~d}$, or $80 \pm 68 \mathrm{~min}$. This time lag, though highly uncertain, is about a factor of 1.5 larger than the time lag found for the $\mathrm{C}$ IV line in Paper I. It is also consistent with the $R_{\mathrm{BLR}}$-luminosity relations of Kaspi et al. (2000, 2005), which suggest that the Balmer line-emitting region is of order light hours in radius in NGC 4395.

In Figure 13 we present the cross-correlation functions of the $B$-band, UV, and X-ray light curves with the optical $V$-band light curve. Note that a negative time lag implies the $V$-band light curve is lagging behind the other reference light curve. No formal time lag is detected between the $B$-band and $V$-band light curves, with a $\mathrm{CCF}$ centroid of $-0.0064_{-0.0076}^{+0.0074} \mathrm{~d}$ (Figure [13 a). Our results suggest a time lag between the UV and optical light curves of $-0.017_{-0.005}^{+0.006} \mathrm{~d}$ (Figure $13 \mathrm{~b}$ ). Within the $1 \sigma$ uncertainty quoted here this is not consistent with zero time lag and implies the optical emission is lagging behind the UV emission by $24_{-9}^{+7} \mathrm{~min}$.

For the cross-correlation of the X-ray and optical light curves, we first used each of the two X-ray visits alone with the simultaneous optical (KAIT) observations and then cross-correlated both visits as one light curve with the corresponding optical light curve. These CCFs are presented in Figures 13 r-e. Cross-correlating only the Xray light curve from Visit 1 with the optical light curve yields a time lag of $-0.032_{-0.009}^{+0.009} \mathrm{~d}$ (Figure 13.). Crosscorrelating only the X-ray light curve from Visit 2 with the optical light curve yields a time lag of $-0.035_{-0.007}^{+0.009}$ $\mathrm{d}$ (Figure 13]). Cross-correlating the X-ray light curve from both visits with the optical light curve yields a time lag of $-0.026_{-0.009}^{+0.012} \mathrm{~d}$ (Figure 13.

We note that due to the shape of the optical light curve during Visit 1, being generally monotonically decreasing, the peak seen in the cross-correlation with the X-ray data (Figure 13) is shallow and broad. In Visit 2 more structure is seen in the optical light curve, resulting in a somewhat better defined peak when cross-correlating the light curves (Figure 13 $\mathrm{d}$ ). The strong and fast variations evident in the X-ray data are not seen in the optical light curve, which also causes the peak cross-correlation coefficient to be relatively small (of order $0.3-0.4$ ). Thus the cross-correlation is more an indication of the general trend of the optical light curve seen in the X-ray light curve. We also note that the average optical flux during Visit 2 was higher than in Visit 1, but in X-rays both visits have about the same flux. This contributes to the very small peak correlation coefficient seen in Figure 13. when cross-correlating the data from both visits at once. Nevertheless, even with the small correlation coefficients, by simply averaging the above three results we can determine that the optical continuum is lagging the $\mathrm{X}$-ray continuum by about $0.031 \pm 0.009 \mathrm{~d}$, or $44 \pm 13 \mathrm{~min}$.

Cross-correlation of the X-ray light curve with the UV light curve is presented in Paper II, where the time lag is found to be $-0.003 \pm 0.016 \mathrm{~d}$, consistent with zero lag, with a $95 \%$ upper limit of roughly $\pm 0.04 \mathrm{~d}$, or \pm 1 hour. We note that the reason for not being able to firmly determine a time lag between the UV and the X-rays is probably because these light curves do not overlap at the end of Visit 2. Thus the strong decrease seen at the end of the UV light curve is not seen in the X-ray light curve. This prevents us from significantly determining a time lag. The large gaps in the UV light curve due to the HST orbit also diminish the possibility of a firm determination of the lag.

Finally, as a consistency check on our observations, we performed cross-correlations between the Lick continuum " $B$ " and " $V$ " light curves (Figures 4 and 5 ) with the simultaneous photometric $B$-band and $V$-band light curves (Figure 2). The bad transit points were removed prior to our calculations. In every case but one, the CCFs were clearly peaked at zero lag (as expected) with correlation coefficients ranging from 0.3 to 0.8 . The one exception was the $V$-band correlation on April 10. The CCF was very broad and flat, but this is easily understood given the gradually, monotonically decreasing light curve in the $V$ band. By comparison, the $B$ band exhibits more structure, and so a lag is more easily computed.

We also performed cross-correlations between the spectroscopically derived broad-band light curves from Figures 9 and 10 with their corresponding photometric light curves. The transit points were again removed. We consider these derived broad-band light curves a much better indicator of the spectroscopic data quality than the individual continuum light curves, because far more flux enters the simulated band pass, increasing the $\mathrm{S} / \mathrm{N}$. Once again, three of the four correlations were clearly consistent with zero lag, with correlation coefficients ranging from 0.4 to 0.8 . The exception was again the $V$-band light curve on April 10. For the same reasons as above, this CCF was very broad and flat and therefore easily understood.

While the Lick spectroscopic data are very noisy, especially in the continuum, these cross-correlation checks above are reassuring. Combined with the KPNO light curves at the end of April 11, we feel confident about the consistency between the various spectroscopic and photometric data sets, and with all our cross-correlations above.

\section{DISCUSSION}

\subsection{Continuum Variability}

Continuum variations in AGNs are generally more pronounced at shorter wavelengths than at longer ones. This is detected in the photometry of NGC 4395, especially between the KAIT and Nickel data on April 11. We see it in the spectra as well, with all variability parameters increasing from $6410 \AA$ to $3650 \AA$. This trend continues into the UV at $1350 \AA$ (Paper I) and into the 0.4-8.0 keV X-rays (Paper II). Finally, the overall continuum 
level shows a larger rise in the UV than in the optical over the course of three months, from April 11 to July 3 ; the $V$-band brightness was 1.5 times larger while the UV was 3.4 times larger.

These variability results are in agreement with the broad picture of AGN variability (Ulrich et al. 1997). Previous studies of NGC 4395, for instance, revealed the continuum level varying by a factor of $\sim 2.2$ at $3800 \AA$ over six months, as compared to a factor of $\sim 1.3$ at $6800 \AA$ (Lira et al. 1999). In NGC 3783, Reichert et al. (1994) report a value of $R_{\max }$ that nearly doubles from $2700 \AA$ to $1460 \AA$. In NGC 4151, Kaspi et al. (1996) found continuum variations of $17 \%-35 \%\left(R_{\max }-1\right)$, increasing from $6925 \AA$ to $4600 \AA$. The trend continues into the near-UV and far-UV. Edelson et al. (1996) also measured $F_{\text {var }}$ values of $24 \%, 9 \%, 5 \%, 4 \%$, and $1 \%$ in the X-ray, at $1275 \AA, 1820 \AA, 2688 \AA$, and $5125 \AA$, respectively. In NGC 5548, one of the best-studied AGNs to date, Korista et al. (1995) found $F_{\mathrm{var}}$ and $R_{\max }$ to be greatly increasing from $5100 \AA$ to $1145 \AA$ during a simultaneous observing campaign. Dietrich et al. (1998) found $F_{\text {var }}$ to nearly double from the $I$ band to the $B$ band in 3C 390.3. Giveon et al. (1999) analyzed $B$-band and $R$-band light curves of $42 \mathrm{PG}$ quasars observed during $\sim$ 30-60 epochs over 7 years. They found a rms variability amplitude of $14 \%$ in $B$ vs. $12 \%$ in $R$. Furthermore the majority of the quasars, and particularly those that were the most variable, were significantly bluer when they were brighter. For example, in all quasars that varied by $\Delta B>0.2 \mathrm{mag}, \Delta B$ was significantly larger than the corresponding $\Delta R$.

As another comparison, Skelton et al. (2005) find significant continuum variability in NGC 4395 of $~ 35 \%$ over the course of 3 nights in 1998. They also find marginal evidence for greater variability in the blue continuum than in the red. Our results appear to be consistent, if at a lower variability amplitude. Skelton et al. also attempted to detect, without success, variability in broad $\mathrm{H} \beta$ by decomposing the line into narrow and broad Gaussian components. We initially attempted the same procedure in all of the lines, in order to isolate the highly variable broad component, but found the results too unreliable. A Gaussian model with one or two components (or four in the case of the $\mathrm{H} \alpha$ complex) is simply an approximation, especially for the broad lines, that may not be uniquely fit. We indeed found some slight inverse correlations between various model components, suggesting an unstable solution to the line profile as a function of time. For this reason we abandoned this method and proceeded with straightforward line integration of all the lines, including single-component narrow lines. This method unfortunately includes non-varying narrow-line flux in the measurement, diminishing the variability amplitude, but is far more reliable.

\subsection{X-ray/UV/Optical Correlations}

Our optical spectroscopy shows hints of a positive lag of the Balmer emission lines behind the optical continuum. The lag is consistent with being roughly a factor of 1.5 longer than for the UV lines and the UV continuum. This is expected, as in most objects studied thus far the time lag for the UV lines (particularly $\mathrm{Ly} \alpha$ ) was always smaller than that found for the Balmer lines, by a factor of about two (e.g., Peterson et al. 2004; Onken \& Peterson 2002; Peterson et al. 1991).

In previous multiwavelength monitoring campaigns of AGNs, the temporal resolutions were typically insufficient to detect any time lag between X-ray, UV, and optical continuum variations. Indeed, in NGC 4151, Edelson et al. (1996) place an upper limit of any X-ray/UV/optical interband delay of $0.15 \mathrm{~d}$, while Kaspi et al. (1996) detect no evidence of a UV-optical continuum lag. Shemmer et al. (2003) find significant evidence for correlated X-ray/optical emission with zero lag (within $1 \mathrm{~d}$ ) in NGC 4051, though their results suggest a very complex relationship between X-ray and optical emission, one that is not simple reprocessing. In the same system, Peterson et al. (2000) also find evidence for correlated X-ray/optical emission, but again the time resolution of their experiment is insufficient to determine a lag of order days or less. With a resolution of $\sim 1 \mathrm{~d}$, Peterson et al. (1991) find no significant lag between the UV and optical continua in NGC 5548. A reanalysis of earlier data results in no UV-optical lag for NGC 5548, NGC 3783, and Fairall 9, while in NGC 7469 there is a UV-optical lag of $\sim 1-2 \mathrm{~d}$ (Peterson et al. 1998; Collier et al. 1998).

Some experiments were successful at measuring an interband delay. For instance, in NGC 4051, Mason et al. (2002) detect the UV emission lagging the X-rays by 0.14 d. In Ark 564, Shemmer et al. (2001) detect the UV continuum lagging the X-rays by $\sim 0.4 \mathrm{~d}$, the optical lagging the UV by $\sim 1.8 \mathrm{~d}$, and the optical lagging the $\mathrm{X}$-rays by $\sim 2 \mathrm{~d}$ (though in general the optical and X-ray light curves are not strongly correlated), all consistent with reprocessing models. By contrast, no correlations at any lag between optical, UV, and X-ray bands in NGC 3516 were found, arguing against the reprocessing model in this case (Maoz et al. 2002; Edelson et al. 2000).

Our results are quite similar, although with large uncertainties, with a UV-optical lag of $24_{-9}^{+7} \mathrm{~min}$ and a Xray-optical lag of $44 \pm 13 \mathrm{~min}$. A high sampling frequency ( $\sim 5$ min observations for optical, $200 \mathrm{~s}$ for UV, $300 \mathrm{~s}$ bins for X-rays) has allowed us to make these sub-hour lag measurements. The $B$-band and $V$-band correlation is consistent with zero, though it hints at a possible $V$ band lag behind the $B$-band. Given the proximity of these two bands, a formal lag of zero is not surprising. Although no formal lag was detected between the UV and $\mathrm{X}$-ray light curves, the fact that the optical continuum lags the X-ray continuum more than the UV continuum is consistent with the idea of X-ray photons being reprocessed into UV and then optical photons. The difference between the X-ray-optical lag and the UV-optical lag is also well within the $95 \%$ upper limit of \pm 1 hour for the X-ray-UV lag established in Paper II.

We can further examine the validity of this model by estimating the energies of the variable components in the three bands. If the X-rays are indeed the driving force behind UV and optical $(V)$ variability, then we must require $\Delta L_{\mathrm{X}} \gtrsim \Delta L_{\mathrm{UV}} \gtrsim \Delta L_{\mathrm{opt}}$ for the model to be energetically reasonable. In Paper II we determined the monochromatic flux variability during Visit 2 was $\sim 2 \times 10^{-13}$ erg $\mathrm{cm}^{-2} \mathrm{~s}^{-1}$ at both $1350 \AA$ and $1 \mathrm{keV}$, using the root of the unnormalized excess variance (i.e., $F_{\text {var }}$ times the mean). For the $V$ band, the unnormalized excess vari- 
ance is roughly $0.023 \times\left(5 \times 10^{-16}\right) \mathrm{erg} \mathrm{cm}^{-2} \mathrm{~s}^{-1} \AA^{-1}$. This corresponds to a monochromatic flux variability at $5500 \AA$ of $\sim 6 \times 10^{-14} \mathrm{erg} \mathrm{cm}^{-2} \mathrm{~s}^{-1}$, which satisfies our energy requirement.

It is also interesting to note that this model for continuum variability extends into the near-infrared $J H K$ bands. Minezaki et al. (2006) provide evidence of nearinfrared intraday variability in NGC 4395 which is correlated with simultaneous $V$-band observations. Furthermore, the near-infrared light curves lag behind the $V$ band light curve by $7.2_{-8.6}^{+15.8} \mathrm{~min}$, which although consistent with zero lag, hints at a possible short duration lag, similar to the $B$ and $V$ bands in this paper.

\subsection{Black Hole Mass Determination}

Unfortunately, due to the very low activity level, low variability amplitude, severe depletion of broad-line flux, and high corresponding noise in the light curves, we are unable to make a significant reverberation-based mass measurement of the SMBH in NGC 4395 to compare with the UV reverberation-based mass of $M_{\mathrm{BH}}=$ $(3.6 \pm 1.1) \times 10^{5} M_{\odot}$ from Paper I. The emission-line light curves for the "variable" lines failed to produce significant time lags behind the continuum light curve, as described in Section [3.3 We are also unable to verify the BLR radius vs. luminosity relationship determined in Paper I.

The lack of significant variability can be seen in the rms spectra (Figures 14 and 15). He II completely disappears in the rms spectra, and broad $\mathrm{H} \beta$ is very weak. The variability in the narrow lines is a result of variable seeing. Since the narrow lines are not spectrally resolved by the instrument and the seeing disk is smaller than the slit width, changes in seeing will alter the line shape as a function of time, while keeping the total integrated flux constant. Because the resolution of the setup we used with the Lick Kast spectrograph is higher on the red side than on the blue side, these line-shape variations are more prominent in the red-side rms spectra. (On the red side, the noise induced by the compromised electronics might also be responsible for the slightly increased rms signal in the narrow lines.) The broad lines are fully resolved, however, so any variability seen in the rms spectra is intrinsic.

The broad component of $\mathrm{H} \alpha$ is diminished and the broad $\mathrm{H} \beta$ practically disappears in the rms spectra. At earlier epochs, the broad He II component was strong and very broad; it was a prime candidate for reverberation mapping. As a qualitative comparison, Figure 16 shows a composite spectrum from 2004 April 10 (total exposure time is 6.58 hours) compared with a single 20min exposure taken on 2003 June 8, both from the 3-m Shane telescope at Lick Observatory. The BLR is noticeably reduced during the low level of activity in the 2004 data, as is the continuum flux. The two spectra show similar $\mathrm{S} / \mathrm{N}$ despite the large difference in exposure times, highlighting the low flux level in the 2004 data. It should be noted that the 2003 data were taken with a $4^{\prime \prime}$ slit, though as this is an essentially bulgeless system, there should be insignificant host-galaxy stellar light (Filippenko \& Ho 2003).

Still, our data do show hints of a possible emission-line lag of $80 \pm 68 \mathrm{~min}$, and it is constructive to make a rough estimate of the mass of the SMBH, to compare with the value derived in Paper I. We estimate the FWHM of the "variable" or broad part of $\mathrm{H} \alpha$, the line exhibiting the greatest variability, to be $\sim 50 \AA$ (or $\sim 2300 \mathrm{~km} \mathrm{~s}^{-1}$ ). While the amplitude of the variability may be suspect due to the electronic difficulties, the lag itself has the greatest significance. Here we assume that the FWHM $\approx \sigma$ for the broad $\mathrm{H} \alpha$ line, as was found for the broad $\mathrm{C}$ IV line in rms spectra (Table 4 in Paper I). While this is a poor approximation for a pure Gaussian line, the broad lines are not strictly Gaussian.

The mass of the $\mathrm{SMBH}$ is given by

$$
M_{\mathrm{BH}}=\frac{f \sigma^{2} c \tau}{G}
$$

where $c$ is the speed of light, $G$ the gravitational constant, and $f$ is a scale factor that depends on the unknown geometry and kinematics of the broad-line region. To be consistent with Paper I, we use the value found empirically by Onken et al. (2004) of $\langle f\rangle=5.5$. Using $\tau \approx 80$ min, we therefore find $M_{\mathrm{BH}} \approx 3 \times 10^{5} M_{\odot}$, which is consistent with our results from Paper I. We stress again that this is simply a qualitative comparison, as our data are of insufficient quality to provide a significant black hole mass determination.

\section{CONCLUSIONS}

We observed NGC 4395, the least luminous known Seyfert 1 galaxy, from five different ground-based optical telescopes over the course of 2 nights in 2004 April, as part of a coordinated multiwavelength reverberationmapping campaign designed to measure the mass of the central black hole. The results of our observations are as follows.

1. NGC 4395 was in a very low state of activity during this period, with optical continuum flux levels, variability amplitudes, and broad emission-line fluxes all diminished compared with earlier epochs.

2. Despite this, we detect intrinsic continuum variability, from both spectra and broad-band photometry, ranging from about $2 \%$ to $10 \%\left(F_{\text {var }}\right)$. In general, we clearly see evidence for increased variability toward shorter wavelengths, consistent with other typical AGNs. This trend extends into the simultaneous UV and X-ray bands. The overall shape of the optical continuum light curves agrees with the simultaneous UV and X-ray light curves. Variability was greatest on April 10, while April 11 exhibited a slightly shallower dependence of variability on wavelength.

3. Cross-correlation of the various continuum light curves suggests that the optical emission lags behind the UV emission by $24_{-9}^{+7} \mathrm{~min}$, and the optical emission lags behind the X-ray emission by $44 \pm 13$ min. There is no formal detection of any lag between the UV and X-ray emission, as described in Paper II, and between the $B$ and $V$ bands. These results are consistent with the reprocessing model, in which X-ray photons are reprocessed into UV and optical photons. Our high sampling frequency has allowed us to make these measurements, which have previously been very difficult to achieve. 
4. We only formally detect a lag between the $\mathrm{H} \alpha$ light curve and the $V$-band light curve. Although all other emission-line light curves are consistent with zero lag, the trend as a whole is quite suggestive. In particular, averaging the Balmer-line correlations with the optical continuum yields an emission-line lag of $80 \pm 68 \mathrm{~min}$ behind the continuum. While not very significant, this result is consistent with the relations of Kaspi et al. (2000) for the size of the BLR. The optical emission-line lag is also about 1.5 times larger than the UV emission-line lag behind the UV continuum. We have seen this behavior in other AGNs, which indicates NGC 4395 is behaving typically, albeit faintly, for an AGN.

5. Using the Balmer emission-line lag and the FWHM of the variable broad-line region, we estimate the mass of the $\mathrm{SMBH}$ to be $M_{\mathrm{BH}} \approx 3 \times 10^{5} M_{\odot}$. Again, this measurement is not very significant, and is meant only to compare with the value of $M_{\mathrm{BH}}=(3.6 \pm 1.1) \times 10^{5} M_{\odot}$ derived in Paper I. The results are consistent.

L.-B.D. would like to thank Ryan Chornock for the many insightful discussions and suggestions that were of great assistance. We also thank C. M. Gaskell for helpful, detailed comments. This research is supported by NASA through HST grant GO-09818 from the Space Telescope Science Institute, which is operated by the Association of Universities for Research in Astronomy, Inc., under NASA Contract NAS5-26555. L.-B.D. is grateful for a Julie-Payette/Doctoral Fellowship from the Natural Sciences and Engineering Research Council of Canada and by the Canadian Space Agency. A.V.F.'s group at UC Berkeley is supported by National Science Foundation (NSF) grant AST-0307894, and by a gift from the TABASGO Foundation. S.K. is supported in part at the Technion by a Zeff Fellowship. A.L. acknowledges support from the Israel Science Foundation (Grant \#1030/04) and from the Norman and Helen Asher Space Research Institute. M.C.B. is supported by a Graduate Fellowship from the NSF. P.M.O. acknowledges financial support from PPARC. KAIT was made possible by generous donations from Sun Microsystems, Inc., the Hewlett-Packard Company, AutoScope Corporation, Lick Observatory, the NSF, the University of California, and the Sylvia \& Jim Katzman Foundation. We thank the staffs at Lick and Kitt Peak National Observatories for their help with the observations.

\section{REFERENCES}

Alexander, T. 1997, in ASSL Vol. 218: Astronomical Time Series, ed. D. Maoz, A. Sternberg, \& E. M. Leibowitz (Dordrecht: Kluwer), 163

Blandford, R. D., \& McKee, C. F. 1982, ApJ, 255, 419

Collier, S. J., et al. 1998, ApJ, 500, 162

Dietrich, M., et al. 1998, ApJS, 115, 185

Edelson, R. A., \& Krolik, J. H. 1988, ApJ, 333, 646

Edelson, R., et al. 2000, ApJ, 534, 180

Edelson, R. A., et al. 1996, ApJ, 470, 364

Ferrarese, L., Pogge, R. W., Peterson, B. M., Merritt, D., Wandel, A., \& Joseph, C. L. 2001, ApJ, 555, L79

Filippenko, A. V. 1982, PASP, 94, 715

Filippenko, A. V., \& Ho, L. C. 2003, ApJ, 588, L13

Filippenko, A. V., Ho, L. C., \& Sargent, W. L. W. 1993, ApJ, 410, L75

Filippenko, A. V., Li, W. D., Treffers, R. R., \& Modjaz, M. 2001, in Small Telescope Astronomy on Global Scales, ed. W. P. Chen, C. Lemme, \& B. Paczyński (San Francisco: ASP), 121

Filippenko, A. V., \& Sargent, W. L. W. 1989, ApJ, 342, L11

Gaskell, C. M., \& Peterson, B. M. 1987, ApJS, 65, 1

Gaskell, C. M., \& Sparke, L. S. 1986, ApJ, 305, 175

Gebhardt, K., et al. 2000, ApJ, 539, L13

Giveon, U., Maoz, D., Kaspi, S., Netzer, H., \& Smith, P. S. 1999, MNRAS, 306, 637

Häring, N., \& Rix, H. 2004, ApJ, 604, L89

Ho, L. C., Filippenko, A. V., \& Sargent, W. L. W. 1997, ApJ, 487, 568

Ho, L. C., \& Ulvestad, J. S. 2001, ApJS, 133, 77

Ho, L. C., et al. 2001, ApJ, 549, L51

Iwasawa, K., Fabian, A. C., Almaini, O., Lira, P., Lawrence, A., Hayashida, K., \& Inoue, H. 2000, MNRAS, 318, 879

Kaspi, S., Maoz, D., Netzer, H., Peterson, B. M., Vestergaard, M., \& Jannuzi, B. T. 2005, ApJ, 629, 61

Kaspi, S., Smith, P. S., Netzer, H., Maoz, D., Jannuzi, B. T., \& Giveon, U. 2000, ApJ, 533, 631

Kaspi, S., et al. 1996, ApJ, 470, 336

Korista, K. T., et al. 1995, ApJS, 97, 285

Laor, A. 1998, ApJ, 505, L83

Li, W., et al. 2001, PASP, 113, 1178

Lira, P., Lawrence, A., O'Brien, P., Johnson, R. A., Terlevich, R.,

\& Bannister, N. 1999, MNRAS, 305, 109

Magorrian, J., et al. 1998, AJ, 115, 2285
Maoz, D., Markowitz, A., Edelson, R., \& Nandra, K. 2002, AJ, 124,1988

Maoz, D., \& Netzer, H. 1989, MNRAS, 236, 21

Maoz, D., Smith, P. S., Jannuzi, B. T., Kaspi, S., \& Netzer, H. 1994, ApJ, 421, 34

Marconi, A., \& Hunt, L. K. 2003, ApJ, 589, L21

Mason, K. O., et al. 2002, ApJ, 580, L117

Miller, J. S., \& Stone, R. P. S. 1993, Lick Observatory Technical Report, 66

Minezaki, T., et al. 2006, ApJ, in press

Moran, E. C., Eracleous, M., Leighly, K. M., Chartas, G., Filippenko, A. V., Ho, L. C., \& Blanco, P. R. 2005, AJ, 129, 2108

Moran, E. C., Filippenko, A. V., Ho, L. C., Shields, J. C., Belloni, T., Comastri, A., Snowden, S. L., \& Sramek, R. A. 1999, PASP, 111,801

Netzer, H., \& Peterson, B. M. 1997, in ASSL Vol. 218: Astronomical Time Series, ed. D. Maoz, A. Sternberg, \& E. M. Leibowitz (Dordrecht: Kluwer), 85

Oke, J. B. 1990, AJ, 99, 1621

O'Neill, P. M., et al. 2006, ApJ, in press

Onken, C. A., Ferrarese, L., Merritt, D., Peterson, B. M., Pogge, R. W., Vestergaard, M., \& Wandel, A. 2004, ApJ, 615, 645

Onken, C. A., \& Peterson, B. M. 2002, ApJ, 572, 746

Peterson, B. M. 1993, PASP, 105, 247

Peterson, B. M., et al. 1991, ApJ, 368, 119

—. 2005, ApJ, 632, 799 (Erratum: 2006, ApJ, 641, 638)

-. 2004, ApJ, 613, 682

-. 2000, ApJ, 542, 161

Peterson, B. M., Wanders, I., Horne, K., Collier, S., Alexander, T., Kaspi, S., \& Maoz, D. 1998, PASP, 110, 660

Reichert, G. A., et al. 1994, ApJ, 425, 582

Rodriguez-Pascual, P. M., et al. 1997, ApJS, 110, 9

Shemmer, O., Uttley, P., Netzer, H., \& McHardy, I. M. 2003, MNRAS, 343, 1341

Shemmer, O., et al. 2001, ApJ, 561, 162

Shih, D. C., Iwasawa, K., \& Fabian, A. C. 2003, MNRAS, 341, 973

Skelton, J. E., Lawrence, A., Pappa, A., Lira, P., \& Almaini, O. 2005, MNRAS, 358, 781

Smith, J. A., et al. 2002, AJ, 123, 2121

Tremaine, S., et al. 2002, ApJ, 574, 740

Ulrich, M.-H., Maraschi, L., \& Urry, C. M. 1997, ARA\&A, 35, 445

White, R. J., \& Peterson, B. M. 1994, PASP, 106, 879 
Wrobel, J. M., Fassnacht, C. D., \& Ho, L. C. 2001, ApJ, 553, L23 
TABLE 1

Broad-Band Continuum

Light CuRves

\begin{tabular}{ccc}
\hline \hline JD-2453105 & $F^{\mathrm{a}}$ & $\sigma^{\mathrm{a}}$ \\
\hline \multicolumn{3}{c}{ Wise $(V$ band $)$} \\
\hline 0.23469 & 0.574 & 0.000 \\
0.23966 & 0.560 & 0.004 \\
0.24307 & 0.569 & 0.004 \\
0.24648 & 0.573 & 0.004 \\
0.24988 & 0.554 & 0.004 \\
\hline
\end{tabular}

Note. - Table 1 is published in its entirety in the electronic edition of the Journal. A portion is shown here for guidance regarding its form and content.

${ }^{\text {a }}$ Flux normalized so that $F=$ 1 corresponds to $V=15.91 \mathrm{mag}$ and $B=16.69 \mathrm{mag}$.

b Data points with relative dates beginning with 85 refer to 2004 July 3 Wise data.

TABLE 2

Variability Parameters - Photometry

\begin{tabular}{|c|c|c|c|c|c|c|c|c|c|}
\hline $\begin{array}{c}\text { Obs. } \\
(1)\end{array}$ & $\begin{array}{c}F_{\mathrm{var}} \\
\text { April } 10 \\
(2)\end{array}$ & $\begin{array}{c}R_{\max } \\
\text { April } 10 \\
\quad(3)\end{array}$ & $\begin{array}{c}A_{\text {peak }} \\
\text { April } 10 \\
(4)\end{array}$ & $\begin{array}{c}F_{\mathrm{var}} \\
\text { April } 11 \\
(5)\end{array}$ & $\begin{array}{c}R_{\max } \\
\text { April } 11 \\
(6)\end{array}$ & $\begin{array}{c}A_{\text {peak }} \\
\text { April } 11 \\
(7)\end{array}$ & $\begin{array}{c}F_{\mathrm{var}} \\
\text { July } 3 \\
(8)\end{array}$ & $\begin{array}{l}R_{\max } \\
\text { July } 3 \\
\quad(9)\end{array}$ & $\begin{array}{c}A_{\text {peak }} \\
\text { July 3 } \\
\text { (10) }\end{array}$ \\
\hline Wise ( $V$ band) & 0.042 & $1.20 \pm 0.01$ & $0.184 \pm 0.011$ & 0.025 & $1.13 \pm 0.01$ & $0.126 \pm 0.005$ & 0.019 & $1.08 \pm 0.01$ & $0.075 \pm 0.010$ \\
\hline Nickel ( $B$ band) & 0.030 & $1.18 \pm 0.02$ & $0.159 \pm 0.017$ & 0.034 & $1.18 \pm 0.02$ & $0.171 \pm 0.020$ & & & \\
\hline
\end{tabular}


TABLE 3

Spectroscopic Continuum Light CuRves

\begin{tabular}{ccc}
\hline \hline JD-2453105 & $F_{\lambda}{ }^{\mathrm{a}}$ & $\sigma^{\mathrm{a}}$ \\
\hline \multicolumn{3}{c}{$3650 \AA$ (Lick) } \\
\hline 0.682 & 0.87 & 0.07 \\
0.690 & 0.91 & 0.06 \\
0.699 & 0.76 & 0.07 \\
0.706 & 0.71 & 0.07 \\
0.714 & 0.67 & 0.06 \\
\hline Note. — Table 3 is pub-
\end{tabular}

lished in its entirety in the electronic edition of the Journal. A portion is shown here for guidance regarding its form and content.

a Flux density in units of $10^{-15} \mathrm{erg} \mathrm{cm}^{-2} \mathrm{~s}^{-1} \AA^{-1}$.

b Suspect transit data points. See text for details.

TABLE 4

SPECTROSCOPIC

EMISSION-LiNE Light Curves

\begin{tabular}{ccc}
\hline \hline JD-2453105 & $F^{\mathrm{a}}$ & $\sigma^{\mathrm{a}}$ \\
\hline [O II $]$ & $\lambda 3727$ (Lick) \\
\hline 0.682 & 0.95 & 0.07 \\
0.690 & 0.96 & 0.08 \\
0.699 & 1.07 & 0.09 \\
0.706 & 0.95 & 0.08 \\
0.714 & 0.96 & 0.07 \\
\hline
\end{tabular}

Note. - Table 4 is published in its entirety in the

electronic edition of the Journal. A portion is shown here for guidance regarding its form and content.

a Flux in arbitrary units, normalized to the mean of each night. 
TABLE 5

Variability Parameters - Spectroscopy (Lick)

\begin{tabular}{|c|c|c|c|c|c|c|}
\hline $\begin{array}{l}\text { Feature } \\
\text { (1) }\end{array}$ & $\begin{array}{c}F_{\text {var }} \\
\text { April } 10 \\
\quad(2)\end{array}$ & $\begin{array}{l}R_{\max } \\
\text { April } 10 \\
\quad(3)\end{array}$ & $\begin{array}{c}A_{\text {peak }} \\
\text { April } 10 \\
\quad(4)\end{array}$ & $\begin{array}{c}F_{\text {var }} \\
\text { April } 11 \\
\quad(5)\end{array}$ & $\begin{array}{l}R_{\max } \\
\text { April } 11 \\
\quad(6)\end{array}$ & $\begin{array}{c}A_{\text {peak }} \\
\text { April } 11 \\
\quad(7)\end{array}$ \\
\hline $\mathrm{H} \gamma^{\mathrm{a}}$ & 0 & $1.51 \pm 0.23$ & $0.41 \pm 0.14$ & 0 & $1.34 \pm 0.25$ & $0.30 \pm 0.18$ \\
\hline He II & 0 & $1.85 \pm 0.51$ & $0.57 \pm 0.22$ & 0 & $1.54 \pm 0.29$ & $0.43 \pm 0.18$ \\
\hline $\mathrm{H} \beta$ & 0.020 & $1.27 \pm 0.11$ & $0.24 \pm 0.08$ & 0 & $1.30 \pm 0.10$ & $0.27 \pm 0.08$ \\
\hline $\mathrm{H} \alpha^{\mathrm{b}}$ & 0.033 & $1.20 \pm 0.02$ & $0.18 \pm 0.02$ & 0.044 & $1.20 \pm 0.03$ & $0.18 \pm 0.03$ \\
\hline $0.4-8.0 \mathrm{keV}^{\mathrm{c}}$ & $0.353 \pm 0.020$ & & & $0.362 \pm 0.024$ & & \\
\hline$F_{\lambda}(1350 \AA)^{\mathrm{d}}$ & & & & 0.138 & $1.77 \pm 0.14$ & $0.59 \pm 0.08$ \\
\hline$F_{\lambda}(3650 \AA)$ & 0.103 & $2.20 \pm 0.51$ & $0.71 \pm 0.25$ & 0 & $2.04 \pm 0.56$ & $0.68 \pm 0.33$ \\
\hline$F_{\lambda}(4200 \AA)$ & 0.097 & $1.50 \pm 0.16$ & $0.40 \pm 0.09$ & 0.050 & $1.35 \pm 0.09$ & $0.30 \pm 0.07$ \\
\hline$F_{\lambda}(4550 \AA)$ & 0.080 & $1.44 \pm 0.09$ & $0.36 \pm 0.06$ & 0.043 & $1.29 \pm 0.07$ & $0.26 \pm 0.06$ \\
\hline$F_{\lambda}(5100 \AA)$ & 0.051 & $1.36 \pm 0.10$ & $0.30 \pm 0.07$ & 0.039 & $1.28 \pm 0.07$ & $0.25 \pm 0.05$ \\
\hline$F_{\lambda}(5650 \AA)$ & 0.043 & $1.25 \pm 0.07$ & $0.22 \pm 0.05$ & 0.034 & $1.33 \pm 0.09$ & $0.29 \pm 0.07$ \\
\hline$F_{\lambda}(6200 \AA)$ & 0.040 & $1.26 \pm 0.05$ & $0.22 \pm 0.03$ & 0.036 & $1.25 \pm 0.05$ & $0.22 \pm 0.04$ \\
\hline$F_{\lambda}(6410 \AA)$ & 0.035 & $1.23 \pm 0.07$ & $0.21 \pm 0.05$ & 0.014 & $1.24 \pm 0.08$ & $0.21 \pm 0.06$ \\
\hline
\end{tabular}

${ }^{\text {a }}$ Includes [O III] $\lambda 4363$.

b Includes [N II] $\lambda \lambda 6548,6583$.

c Chandra Visits 1 and 2 (Paper II). The parameters are calculated for the full duration of each observation $(\sim 30 \mathrm{ks})$ in $300 \mathrm{~s}$ bins.

d HST Visit 2 (Paper I).

TABLE 6

Spectroscopically

Derived Broad-Band

Light Curves

\begin{tabular}{ccc}
\hline \hline JD-2453105 & $F^{\mathrm{a}}$ & $\sigma^{\mathrm{a}}$ \\
\hline \multicolumn{3}{c}{ Lick $B$ band } \\
\hline 0.682 & 1.02 & 0.01 \\
0.690 & 1.04 & 0.01 \\
0.699 & 1.07 & 0.01 \\
0.706 & 1.05 & 0.01 \\
0.714 & 1.03 & 0.01 \\
\hline
\end{tabular}

Note. - Table 6 is published in its entirety in the electronic edition of the Journal. A portion is shown here for guidance regarding its form and content.

a Flux in arbitrary units, normalized to the mean of each night.

${ }^{\mathrm{b}}$ Suspect transit data points. See text for details. 


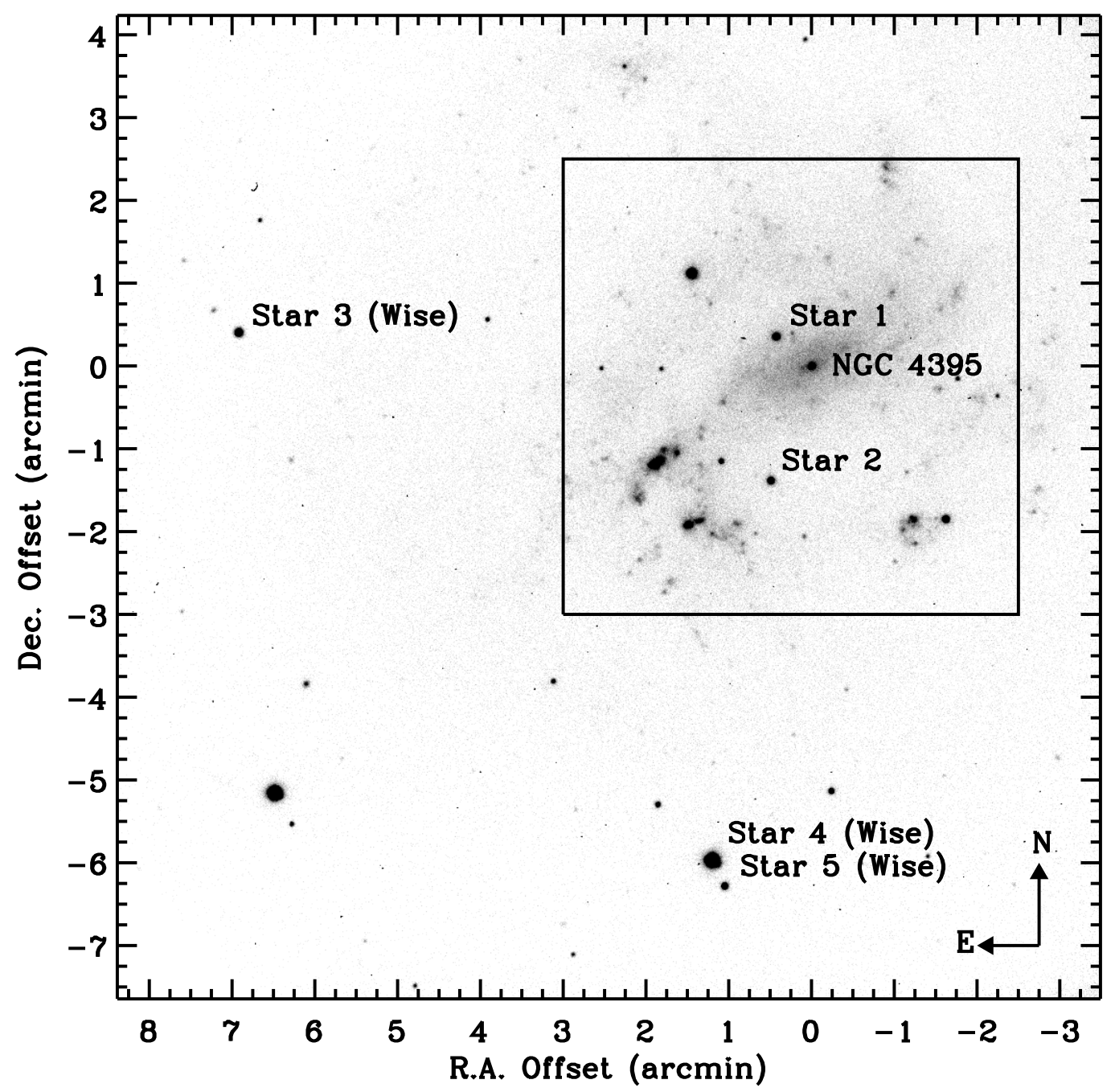

FIG. 1. - The NGC 4395 field (from the Wise Observatory). The KAIT and Nickel field is shown as the inner box. The reference stars are labeled. Stars 1 and 2 are common to all three data sets. 


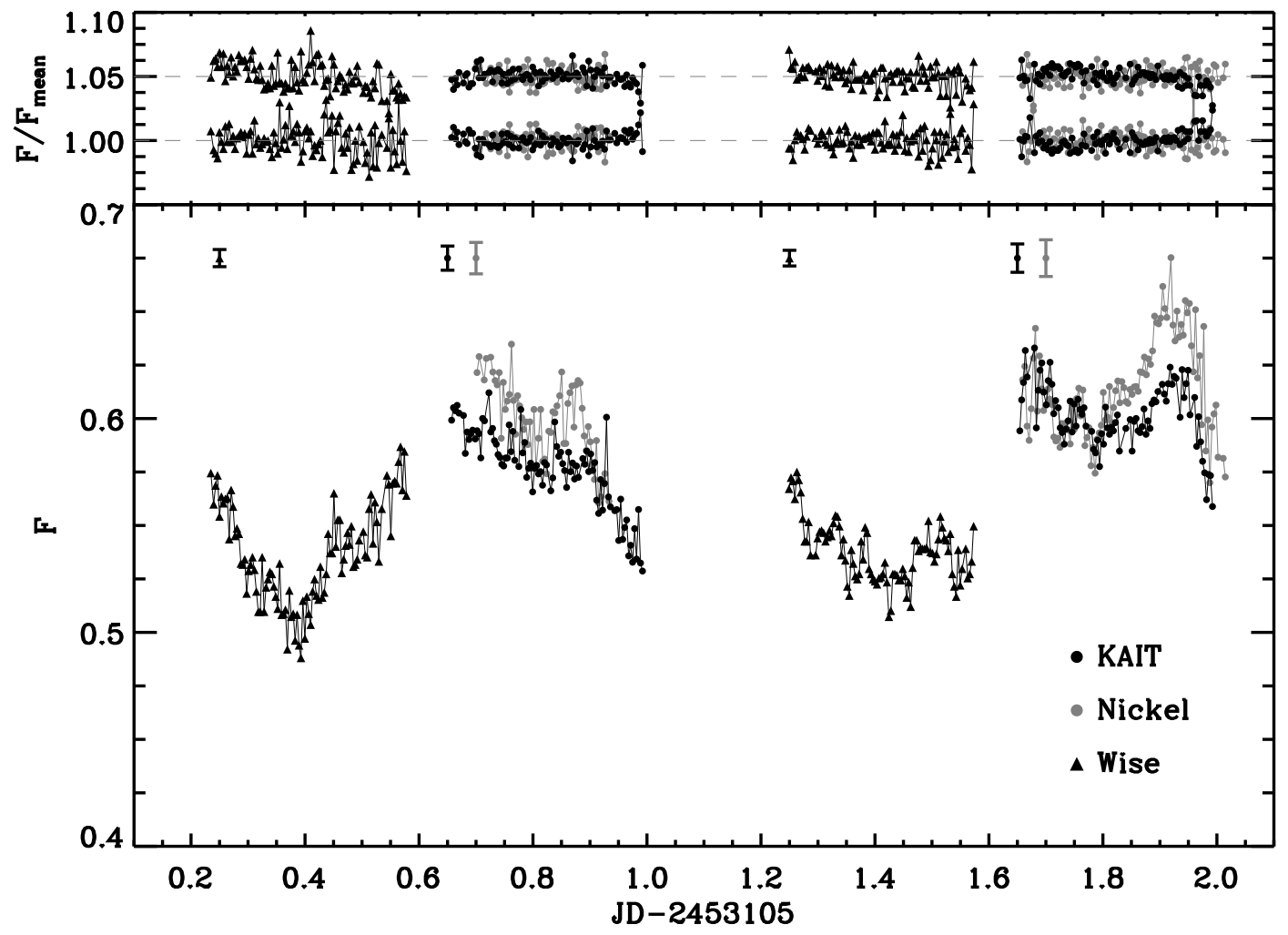

FIG. 2.- Light curves from Wise ( $V$ band), KAIT ( $V$ band), and Nickel ( $B$ band) photometry, 2004 April 10 and 11. The top panel shows, from top to bottom, stars 1 and 2 (from Figure 1), divided by their mean for each night (plus an offset in the case of star 1 , for clarity). The bottom panel shows NGC 4395, normalized to the mean flux of stars 1 and 2 . Flux is normalized such that $F=1$ corresponds to $V=15.91 \mathrm{mag}$ and $B=16.69 \mathrm{mag}$. Typical uncertainties are shown for reference above the light curves. 


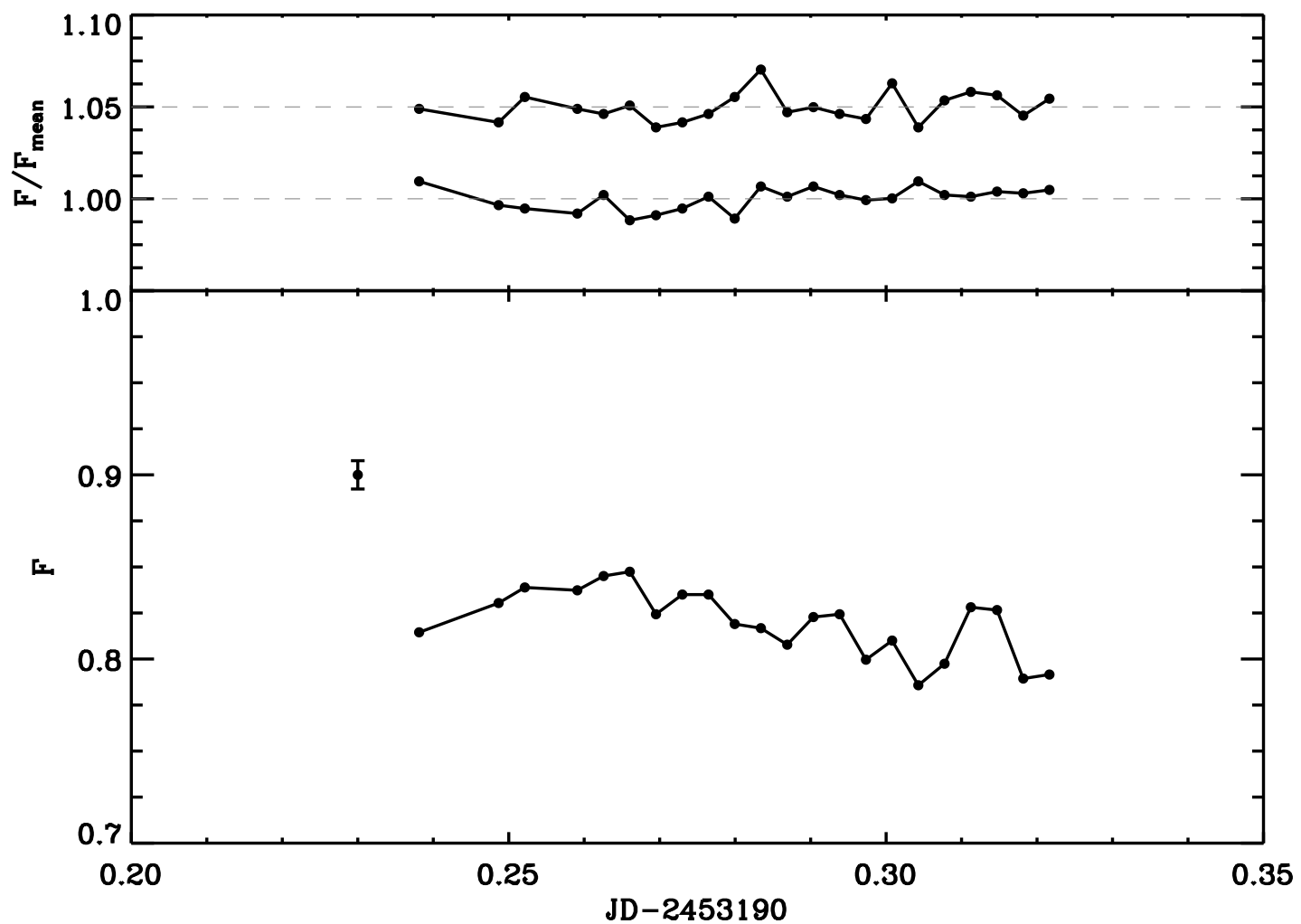

FIG. 3.- Light curve from Wise ( $V$ band) photometry, 2004 July 3. The top panel is as described in Figure 2 The bottom panel shows NGC 4395, normalized in the same way as in Figure 2 with $F$ in the same units. The typical uncertainty is shown for reference above the light curve. 


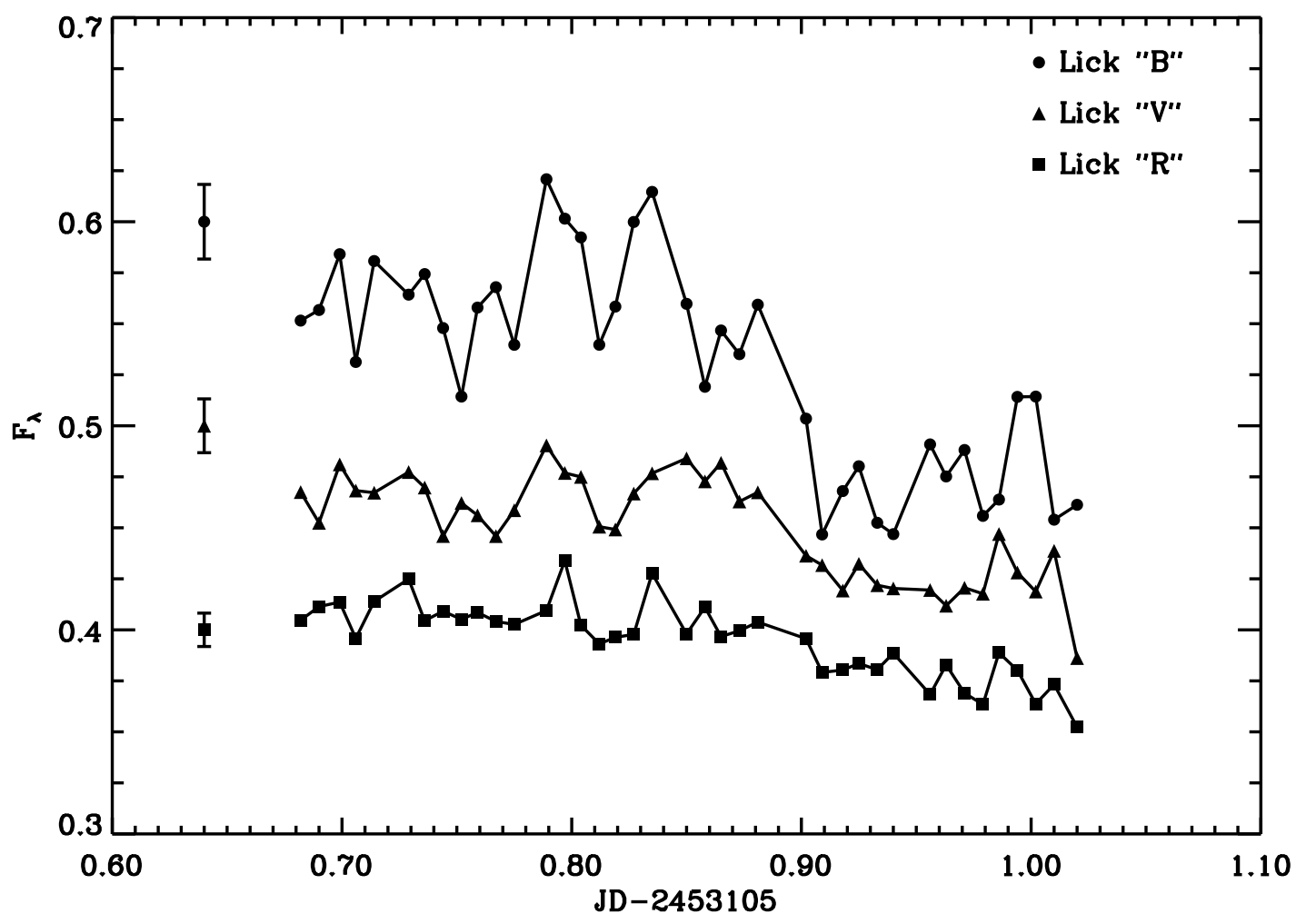

FIG. 4.- Continuum light curves from the Lick data, April 10. Typical uncertainties are shown at left. The $3650 \AA$ continuum measurement is extremely noisy and omitted for clarity. The remaining measurements have been paired and averaged to reduce the noise. The Lick " $B$ " band (circles) is an average of the $4200 \AA$ and $4550 \AA$ continuum measurements; the Lick " $V$ " band (triangles) is an average of the $5100 \AA$ and $5625 \AA$ continuum measurements; the Lick " $R$ " band (squares) is an average of the $6200 \AA$ and $6410 \AA$ continuum measurements. $F_{\lambda}$ is in units of $10^{-15} \mathrm{erg} \mathrm{cm}^{-2} \mathrm{~s}^{-1} \AA^{-1}$. 


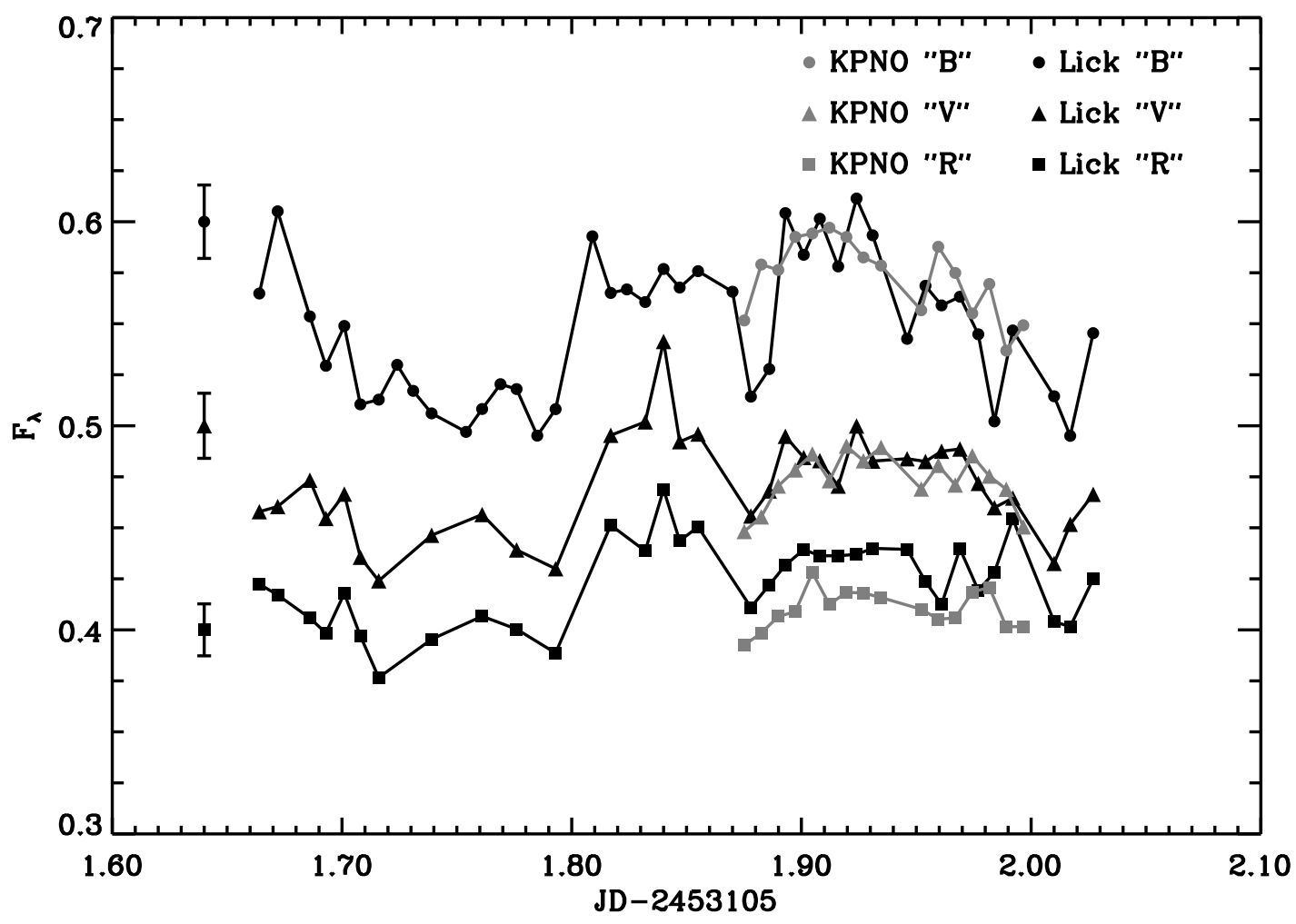

Fig. 5.- Continuum light curves from the Lick data, April 11. Typical uncertainties are shown at left. The Lick " $B$," " $V$," and " $R$ " bands are as described in Fig. 4 KPNO data are also included as gray symbols, with the " $B$," " $V$," and " $R$ " bands having the same definitions as the Lick data. Uncertainties are typically a factor of $\sim 3$ smaller than in the Lick data, and are not shown for clarity. $F_{\lambda}$ is in units of $10^{-15} \mathrm{erg} \mathrm{cm}^{-2} \mathrm{~s}^{-1} \AA^{-1}$. 


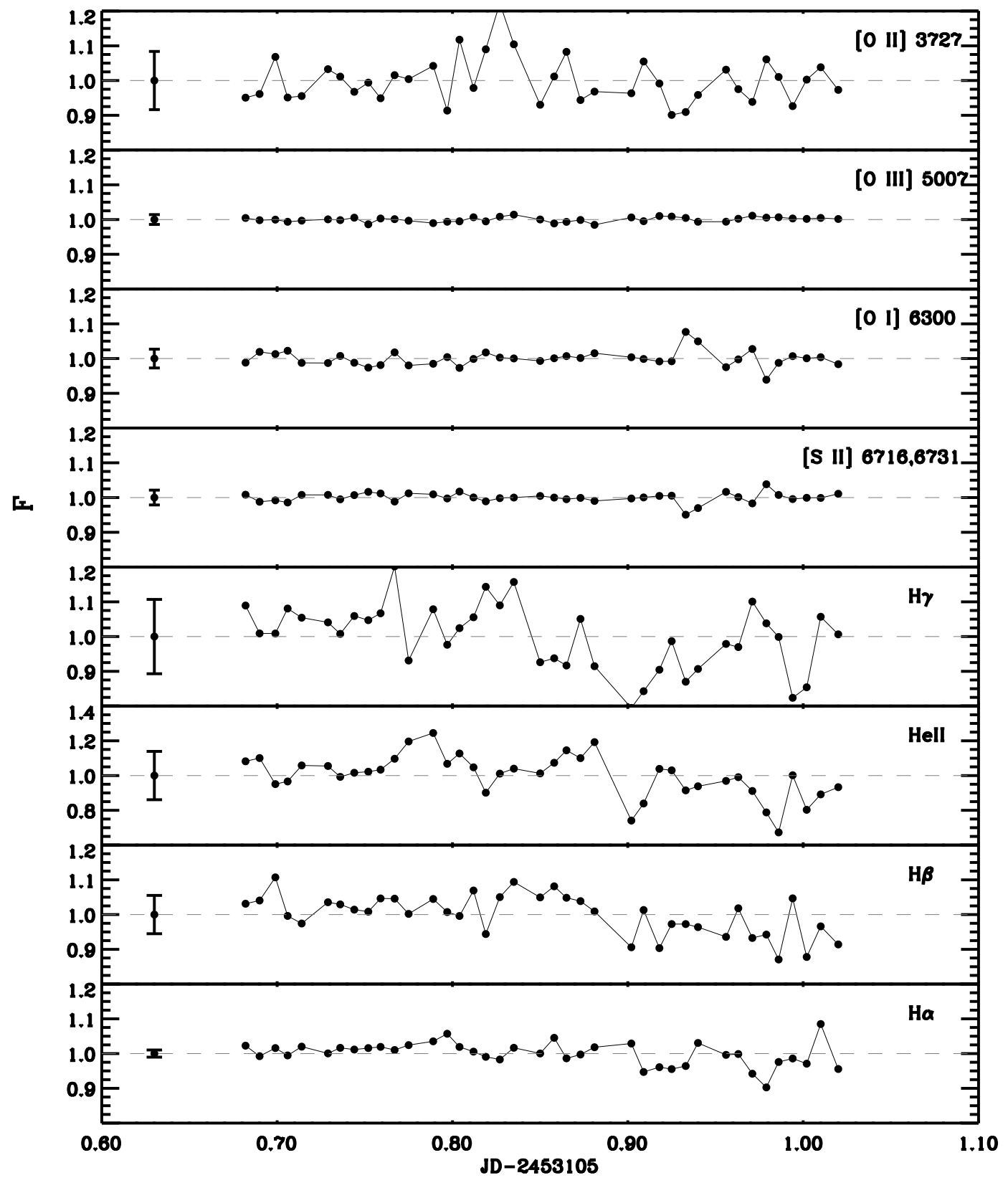

FIG. 6. - Emission-line light curves from the Lick data, April 10. Typical uncertainties are shown on the left of each panel. Note that $[\mathrm{O} \mathrm{I}]$ and $[\mathrm{S} \mathrm{II}]$ are mirrored light curves, as their total flux is assumed to be constant. All light curves are normalized relative to their means; $F$ is therefore in arbitrary units. 


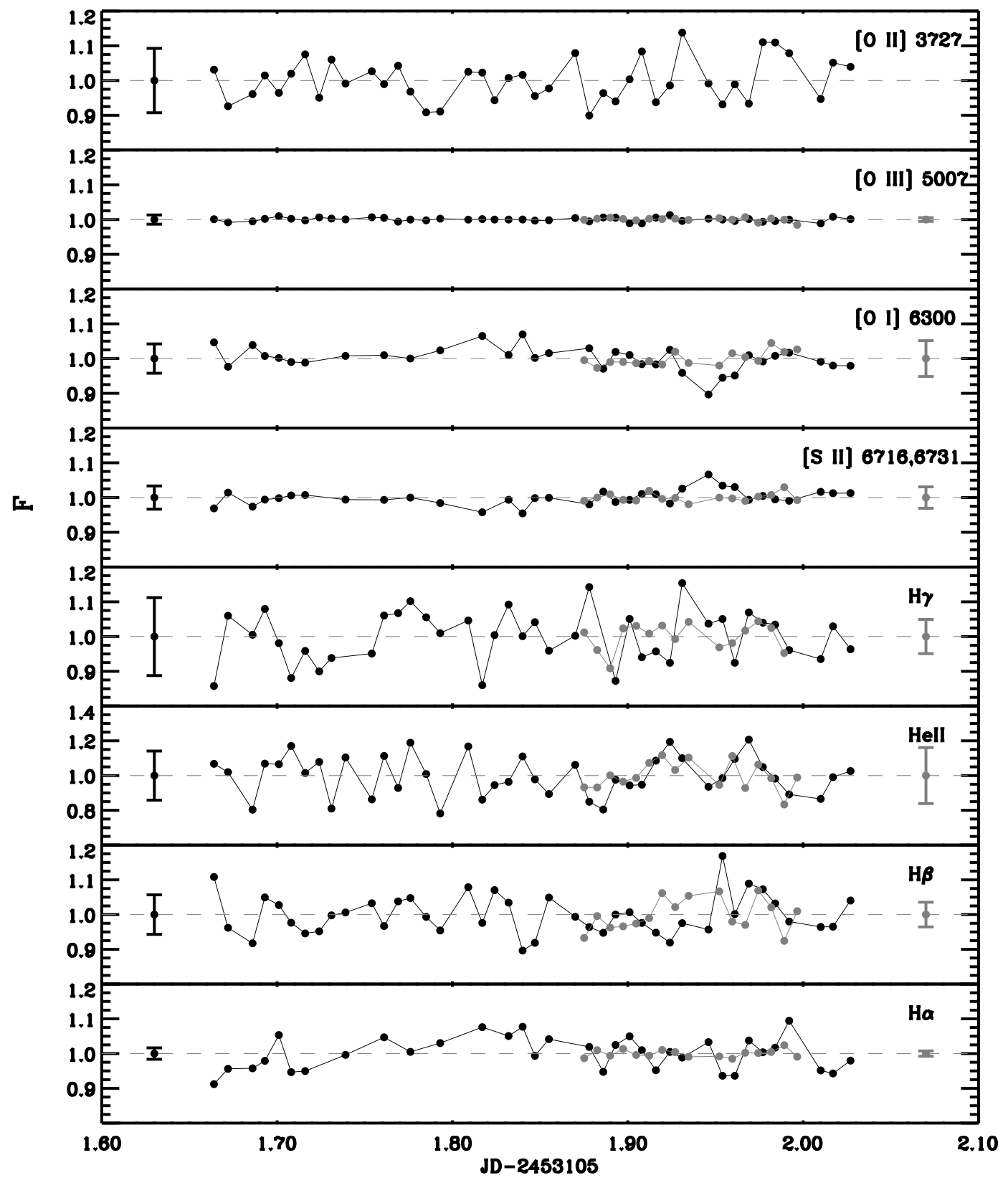

FIG. 7.- Emission-line light curves from the Lick data (KPNO data in gray), April 11. Typical uncertainties are shown on the left (right) of each panel for Lick (KPNO) data. Note that [O I] and [S II] in the Lick data are mirrored light curves, as their total flux is assumed to be constant. All light curves are normalized relative to their means; $F$ is therefore in arbitrary units. 


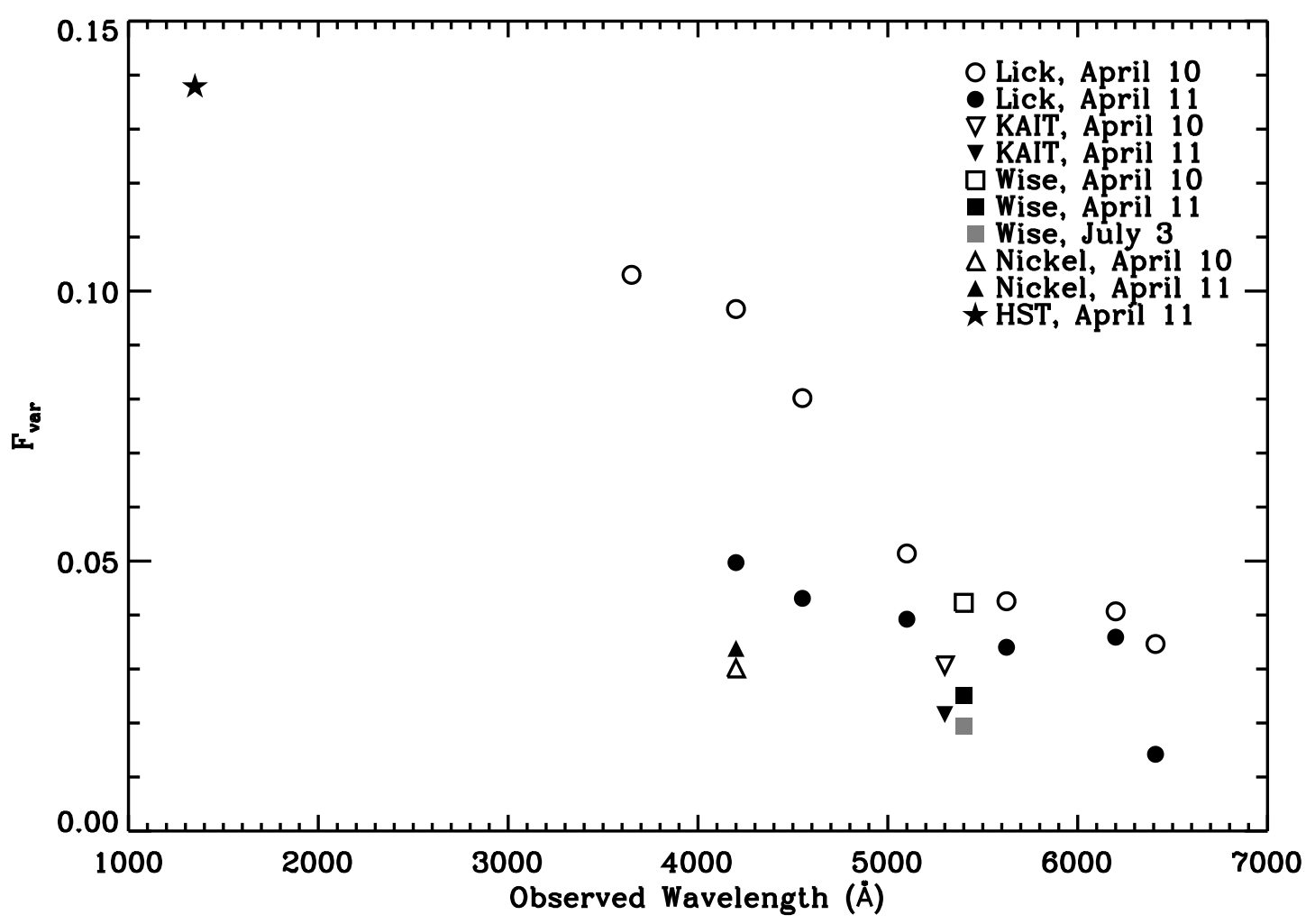

FIG. 8.- Various $F_{\text {var }}$ measurements as a function of wavelength, from Tables 2 and 5 The "Lick" points are spectroscopic continuum measurements. The HST point is from Visit 2, Paper I. The broad-band measurements are placed at the peak of the bandpass, with KAIT and Wise offset slightly from one another for clarity. Not included are the values for the $0.4-8.0 \mathrm{keV}$ X-rays from Paper II, which are 0.353 \pm 0.020 and $0.362 \pm 0.024$ on April 10 and 11 , respectively. The important results are (a) the broad trend of increasing variability with decreasing wavelength, (b) the lower overall variability on April 11, and (c) the shallower dependence of variability on wavelength on April 11 . 


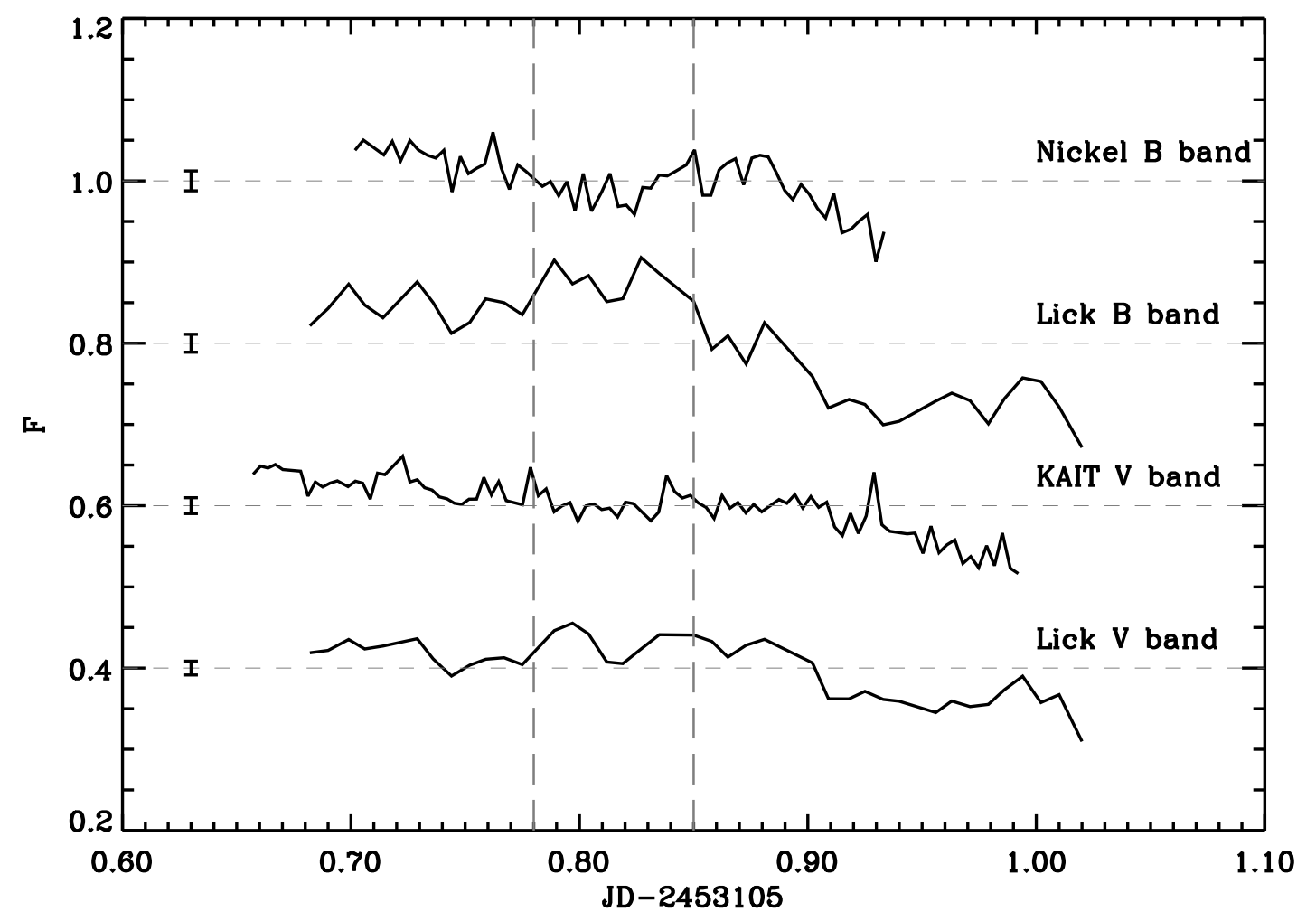

FIG. 9.- Comparison between Lick, KAIT ( $V$ band), and Nickel ( $B$ band) data on April 10. KAIT and Nickel data are as described in Fig. 2 Lick spectra have passed through $B$ and $V$ filter response functions and integrated to create simulated $B$-band and $V$-band observations. As noted in the text, the Lick continuum measurements are likely not as reliable as the KAIT and Nickel measurements, especially during the interval between the vertical dashed lines. Typical uncertainties are shown at left. All light curves are normalized relative to their means and shifted vertically for clarity; $F$ is therefore in arbitrary units. 


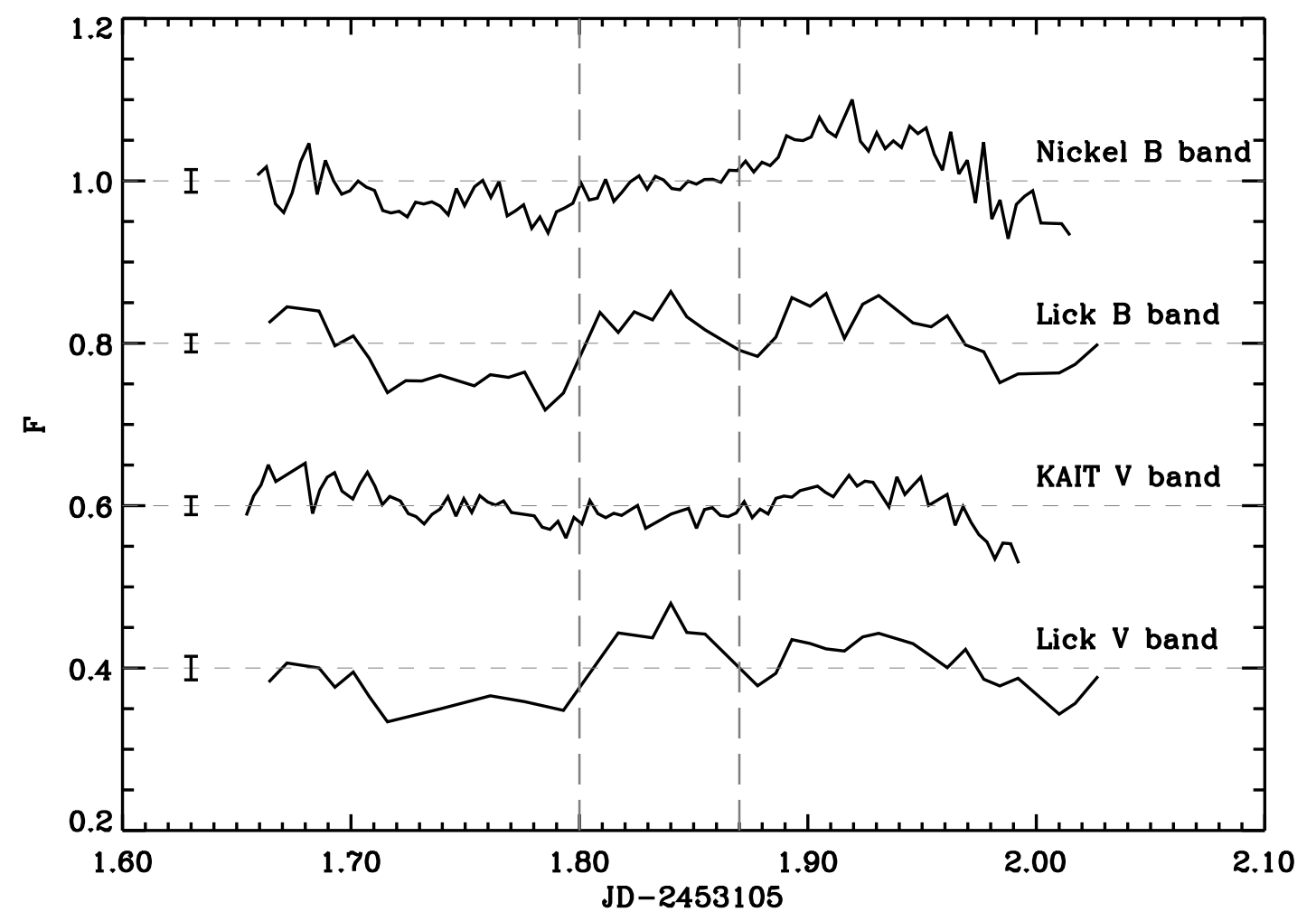

FIG. 10.- Comparison between Lick, KAIT ( $V$ band), and Nickel ( $B$ band) data on April 11. Light curves and vertical dashed lines are as described in Fig. [9 Typical uncertainties are shown at left. All light curves are normalized relative to their means and shifted vertically for clarity; $F$ is therefore in arbitrary units. 


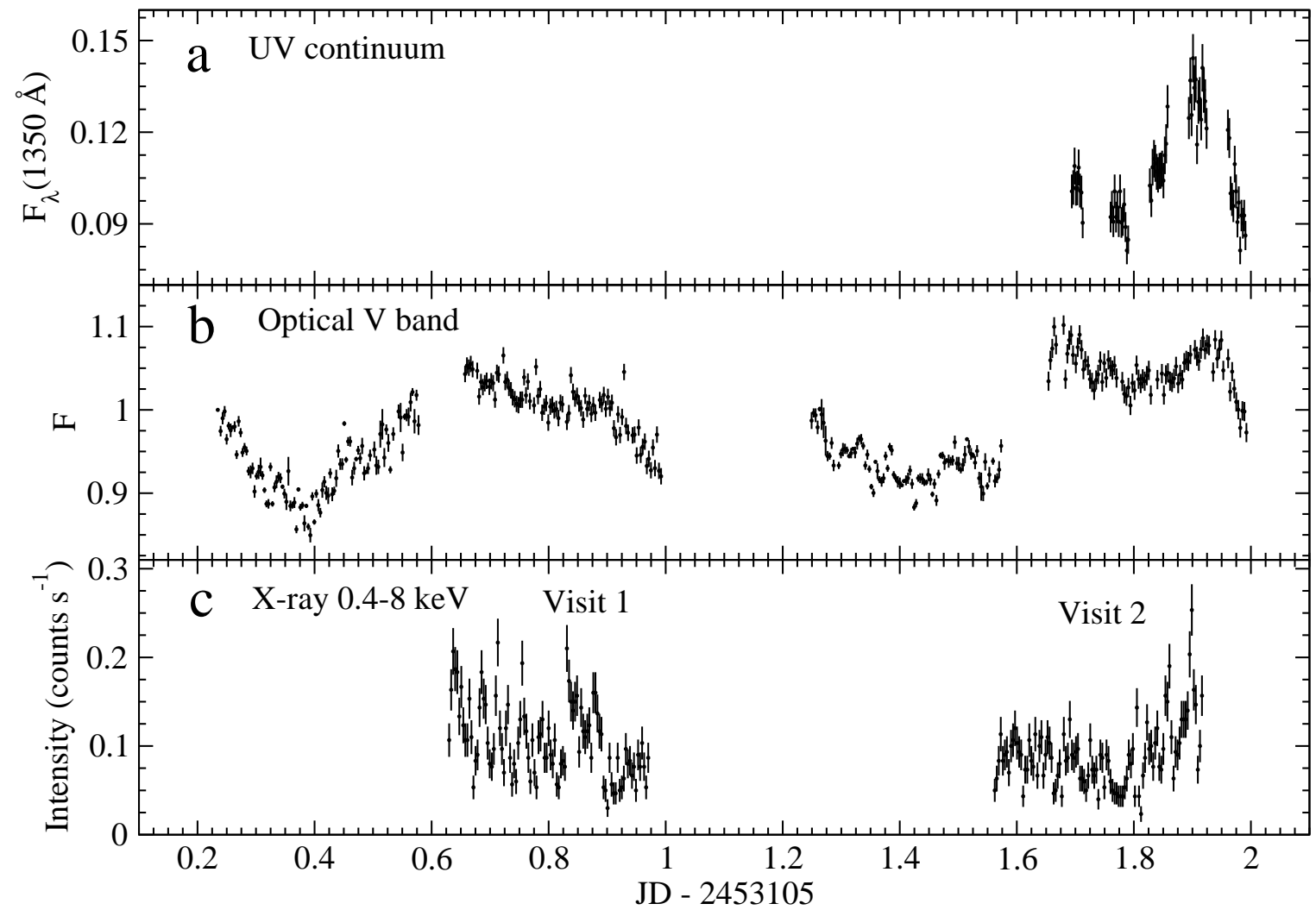

FIG. 11. - Light curves of NGC 4395 in the three bands used for the cross-correlation analysis in this paper. (a) UV continuum at 1350 $\AA$ binned to $200 \mathrm{~s}$. Flux in units of $10^{-14} \mathrm{erg} \mathrm{cm}^{-2} \mathrm{~s}^{-1} \AA^{-1}$ (Peterson et al. 2005). (b) $V$-band light curve from Figure 2 binned to $\sim 300$ s. Flux in arbitrary units. (c) X-ray light curves presented in the top panels of Figure 1 and 2 of O'Neill et al. (2006) binned to $300 \mathrm{~s}$. 


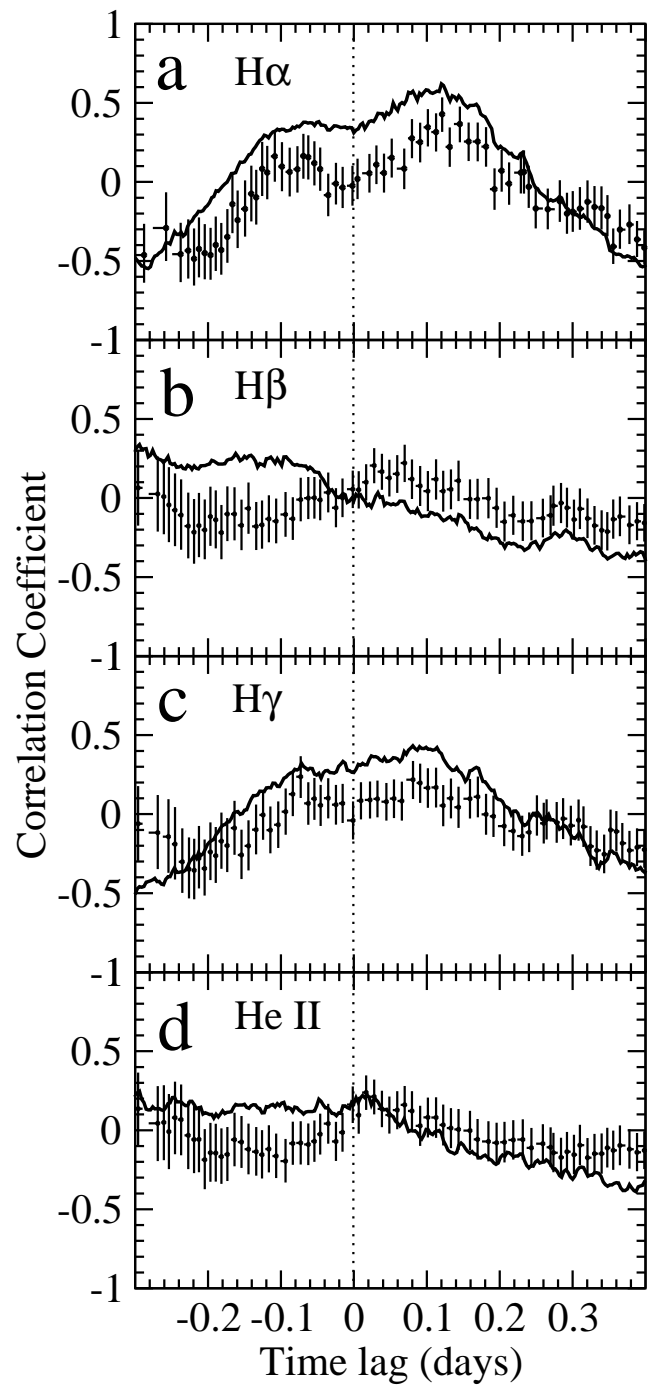

FIG. 12. - CCFs of the optical-line light curves from Figures 6 and 7 with the optical $V$-band light curve (Figure 11p). We present results from the ICCF method (solid line) and from the ZDCF method (points with error bars). See text for details about comparing these two methods. (a) Cross-correlation of the $\mathrm{H} \alpha$ light curve with the optical light curve. (b) Cross-correlation of the $\mathrm{H} \beta$ light curve with the optical light curve. (c) Cross-correlation of the $\mathrm{H} \gamma$ light curve with the optical light curve. (d) Cross-correlation of the He II $\lambda 4686$ light curve with the optical light curve. 


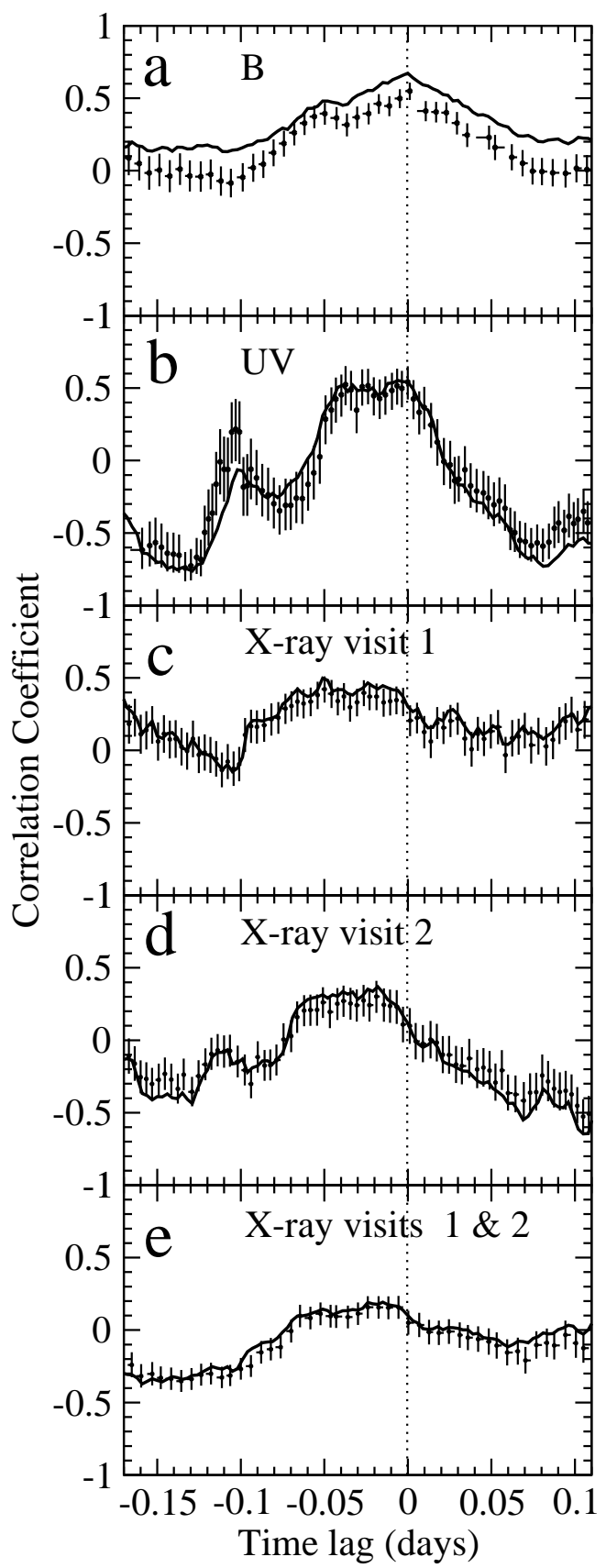

FIG. 13. - CCFs of the B-band, UV, and X-ray continuum light curves with the optical $V$-band light curve, as presented in Figure 11 We present results from the ICCF method (solid line) and from the ZDCF method (points with error bars). The results from both methods are consistent. See text for details. (a) Cross correlation of the $B$-band light curve (Figure 2) with the $V$-band light curve (Figure 11 b). (b) Cross-correlation of the UV light curve (Figure 11]) with the optical light curve (Figure 110). (c) Cross-correlation of the X-ray data from Visit 1 only (Figure 11, left part) with the simultaneous optical light curves (Figure 11b left part). (d) Cross-correlation of the X-ray data from Visit 2 only (Figure 11] right part) with the simultaneous optical light curves (Figure 11p right part). (e) Cross-correlation of the X-ray data from Visits $1 \& 2$ (Figure 117) with the simultaneous optical light curve (Figure 111). 


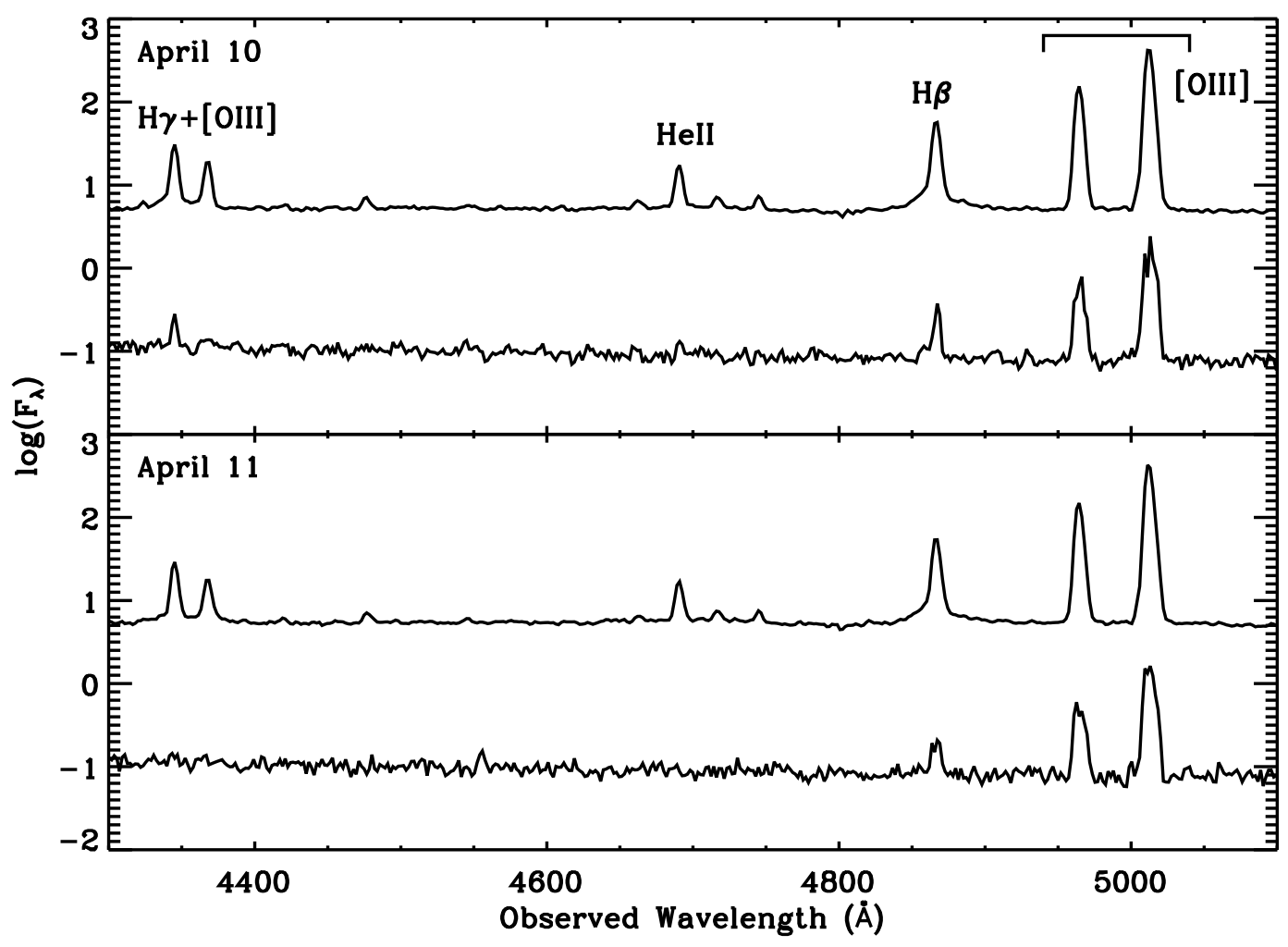

FIG. 14.- Mean and rms blue-side spectra from Lick Observatory. Mean spectra are plotted above the rms spectra. Mean spectra have been shifted up by 1 dex for clarity. Overall variability is low, especially in the broad-line regions. Variable seeing contributes to the narrow lines in the rms spectra. $F_{\lambda}$ is in units of $10^{-15} \mathrm{erg} \mathrm{cm}^{-2} \mathrm{~s}^{-1} \AA^{-1}$. 


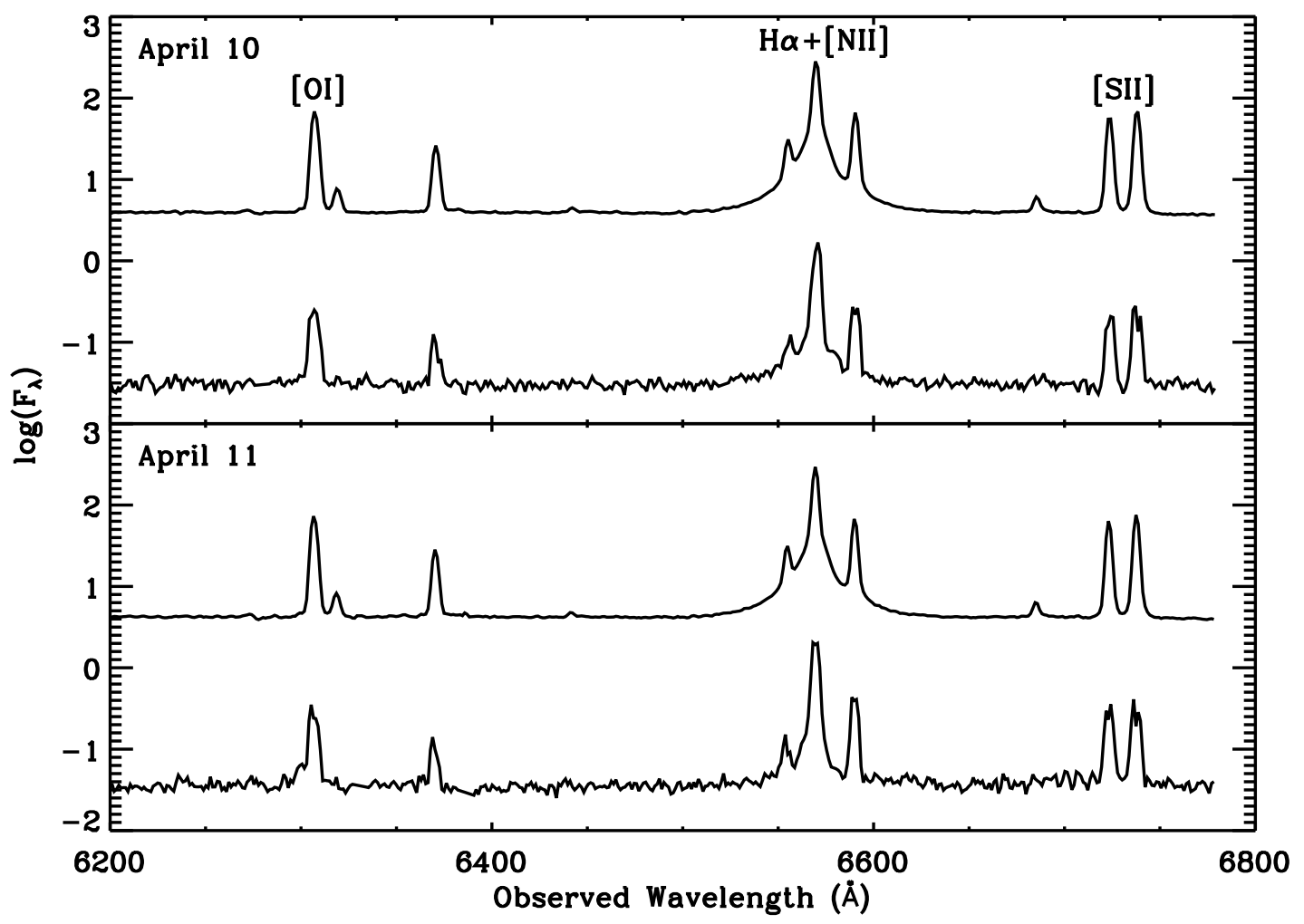

FIG. 15.- Mean and rms red-side spectra from Lick Observatory. Mean spectra are plotted above the rms spectra. Mean spectra have been shifted up by 1 dex for clarity. Overall variability is low. Variable seeing contributes to the narrow lines in the rms spectra. $F_{\lambda}$ is in units of $10^{-15} \mathrm{erg} \mathrm{cm}^{-2} \mathrm{~s}^{-1} \AA^{-1}$. 


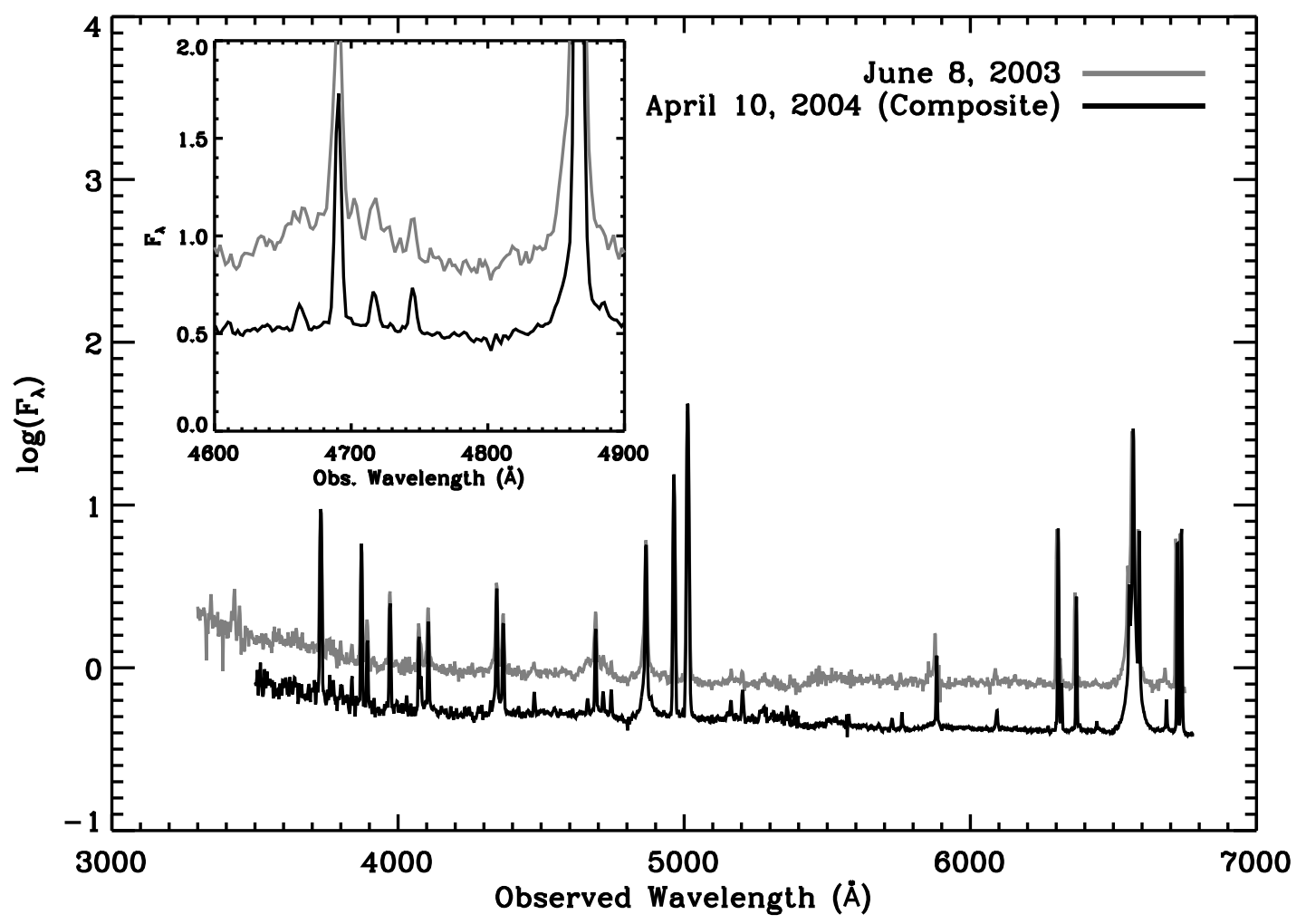

FIG. 16.- Comparison between 2004 April 10 data and 2003 June 8 data, both obtained at Lick Observatory. Data have been scaled so that [O III $\lambda 5007$ fluxes are equal. The inset is a blowup of the He II and $\mathrm{H} \beta$ region. The June 8 spectrum is a single 20 -min exposure, while the April 10 composite spectrum has a total exposure time of $6.58 \mathrm{hr}$. The latter is clearly at a lower level: the flux level is lower, the noise (per exposure time) is higher, and the broad-line components are diminished (especially He II). $F_{\lambda}$ is in units of $10^{-15} \mathrm{erg} \mathrm{cm}^{-2} \mathrm{~s}^{-1} \AA^{-1}$. 